MATHEMATICS OF COMPUTATION

Volume 73, Number 246, Pages 777-812

S $0025-5718(03) 01551-5$

Article electronically published on June 18, 2003

\title{
OPTIMAL RATE OF CONVERGENCE OF A STOCHASTIC PARTICLE METHOD TO SOLUTIONS OF 1D VISCOUS SCALAR CONSERVATION LAWS
}

\author{
MIREILLE BOSSY
}

\begin{abstract}
This article presents the analysis of the rate of convergence of a stochastic particle method for $1 \mathrm{D}$ viscous scalar conservation laws. The convergence rate result is $\mathcal{O}(\Delta t+1 / \sqrt{N})$, where $N$ is the number of numerical particles and $\Delta t$ is the time step of the first order Euler scheme applied to the dynamic of the interacting particles.
\end{abstract}

\section{INTRODUCTION}

We consider the following one-dimensional viscous scalar conservation law:

$$
\left\{\begin{array}{l}
\frac{\partial V}{\partial t}(t, x)=\frac{\sigma^{2}}{2} \frac{\partial^{2} V}{\partial x^{2}}(t, x)-\frac{\partial}{\partial x} A(V(t, x)), \forall(t, x) \in(0, T] \times \mathbb{R}, \\
V(0, x)=V_{0}(x), \forall x \in \mathbb{R} .
\end{array}\right.
$$

We assume that $A: \mathbb{R} \rightarrow \mathbb{R}$ is a $C^{3}$ function and $\sigma>0$. In this article, we analyze the rate of convergence of a stochastic particle method for the numerical solution of (1.1), when the initial condition $V_{0}$ is the cumulative distribution function of a probability measure on $\mathbb{R}$.

When $A(v)=v^{2} / 2$, the conservation law (1.1) is the viscous Burgers equation

$$
\left\{\begin{array}{l}
\frac{\partial V}{\partial t}(t, x)=\frac{\sigma^{2}}{2} \frac{\partial^{2} V}{\partial x^{2}}(t, x)-V(t, x) \frac{\partial V}{\partial x}(t, x),(t, x) \in(0, T] \times \mathbb{R}, \\
V(0, x)=V_{0}(x), \forall x \in \mathbb{R} .
\end{array}\right.
$$

A previous work proposes a stochastic particle method for the numerical solution of the Burgers equation (see Bossy and Talay [2, 3]). The method is based upon the probabilistic interpretation of the Burgers equation as the evolution equation of the cumulative distribution function of a stochastic nonlinear process (in the sense of McKean). The algorithm is inspired by a propagation of chaos result for the system of interacting particles associated with the nonlinear process. Under suitable hypotheses on the initial data $V_{0}$, we proved a convergence rate of order $\mathcal{O}(1 / \sqrt{N}+\sqrt{\Delta t})$ for the $L^{1}(\mathbb{R} \times \Omega)$ norm of the error. $N$ is the number of simulated interacting particles and $\Delta t$ is the time step of the discretization by the Euler scheme of the stochastic differential system that governs the particles motion.

Received by the editor April 5, 2001 and, in revised form, July 30, 2002.

2000 Mathematics Subject Classification. Primary 65C35, 65M15, 60H10, 60K35.

Key words and phrases. Stochastic particle method, viscous scalar conservation laws, Euler discretization scheme, weak convergence rate. 
For the Burgers case, numerical experiments confirm the order $\mathcal{O}(1 / \sqrt{N})$ for the dependence on $N$, but suggest that the dependence in $\Delta t$ is of order $\mathcal{O}(\Delta t)$ rather than $\mathcal{O}(\sqrt{\Delta t})$ (see [3, 1] $)$. In this previous work, we used estimates on the rate of convergence in $L^{2}(\Omega)$ for the Euler scheme whereas, in this sort of numerical computation, the averaging effect due to the propagation of chaos phenomena suggests that we should analyze the discretization error with estimates on the weak rate of convergence for the Euler scheme.

In this article, we extend the stochastic particle method for the Burgers equation in the general context of the viscous scalar conservation law (1.1) and we prove a theoretical rate of convergence of order $\mathcal{O}(1 / \sqrt{N}+\Delta t)$.

To construct the algorithm, we follow Jourdain [6] who gives a probabilistic interpretation of nonlinear parabolic PDEs such as the viscous scalar conservation law (1.1), when $A$ is a $C^{1}$ function and $V_{0}$ is a nonconstant function with bounded variation. In order to lighten the presentation of the algorithm and the convergence analysis, we restrict ourselves to the case of an initial condition $V_{0}(x)=m_{0}((-\infty, x])=H * m_{0}(x)$, where $m_{0}$ is a probability measure on $\mathbb{R}$ and $H(x)=\mathbb{1}_{\{x \geq 0\}}$ denotes the Heaviside function.

In [6], Jourdain provides a natural way to connect (1.1) with a nonlinear martingale problem and proves a propagation of chaos result for the suitable system of weakly interacting particles. Here, we briefly present the main ideas of the probabilistic interpretation of (1.1), when $V_{0}(x)=H * m_{0}(x)$ and $m_{0}$ is a probability measure, as well as some results in [6] on which we base our numerical algorithm: for a probability measure $P$ on $C([0,+\infty), \mathbb{R})$, we define the flow $\left(P_{t}\right)_{t \geq 0}$ of probability measures on $\mathbb{R}$ by $P_{t}=P \circ X_{t}^{-1}$, where $X$ denotes the canonical process on $C([0,+\infty), \mathbb{R})$. Let $C_{b}^{2}(\mathbb{R})$ be the set of bounded functions with bounded first and second order derivatives. We associate to (1.1) the following martingale problem:

Definition 1.1. The probability measure $P \in \mathcal{P}(C([0,+\infty), \mathbb{R}))$ is a solution of the nonlinear martingale problem $\mathcal{M}$, starting at $m_{0}$, if $P_{0}=m_{0}$ and

$$
\begin{aligned}
& \phi\left(X_{t}\right)-\phi\left(X_{0}\right)-\int_{0}^{t}\left(\frac{\sigma^{2}}{2} \phi^{\prime \prime}\left(X_{s}\right)+A^{\prime}\left(H * P_{s}\left(X_{s}\right)\right) \phi^{\prime}\left(X_{s}\right)\right) d s \\
& \text { is a } P \text {-martingale, for any } \phi \in C_{b}^{2}(\mathbb{R}) .
\end{aligned}
$$

We define a system of $N$ particles in mean field interaction by the following stochastic differential equation:

$$
\begin{aligned}
X_{t}^{i, N} & =X_{0}^{i, N}+\sigma W_{t}^{i}+\int_{0}^{t} A^{\prime}\left(\frac{1}{N} \sum_{j=1}^{N} H\left(X_{s}^{i, N}-X_{s}^{j, N}\right)\right) d s, \\
& =X_{0}^{i, N}+\sigma W_{t}^{i}+\int_{0}^{t} A^{\prime}\left(H * \mu_{s}^{N}\left(X_{s}^{i, N}\right)\right) d s, \quad 1 \leq i \leq N,
\end{aligned}
$$

where $\mu^{N}=\frac{1}{N} \sum_{i=1}^{N} \delta_{X^{i, N}}$ is the empirical measure of the particles and $\left(W^{1}, \ldots\right.$, $W^{N}$ ) is an $N$-dimensional Brownian motion independent of the initial variables $\left(X_{0}^{1, N}, \ldots, X_{0}^{N, N}\right)$ which are i.i.d. with law $m_{0}$.

Proposition 1.2 (Jourdain [6]). The martingale problem $\mathcal{M}$ starting at $m_{0}$ admits a unique solution $P$ and the particle systems $\left(X^{1, N}, \ldots, X^{N, N}\right)$ are P-chaotic; that is, for a fixed $j \in \mathbb{N}^{*}$, the law of $\left(X^{1, N}, \ldots, X^{j, N}\right)$ converges weakly to $P^{\otimes} j$ as $N \longrightarrow+\infty$. Moreover, (1.1) has a unique bounded weak solution given by $V(t, x)=$ $H * P_{t}(x)$. 
The propagation of chaos result implies that the empirical cumulative distribution function $H * \mu_{t}^{N}(x)=\frac{1}{N} \sum_{i=1}^{N} H\left(x-X_{t}^{i, N}\right)$ of the particle system at time $t$ converges in $L^{1}(\Omega)$ to the weak solution $V(t, x)$ of (1.1) (see [6]). In practice, the $X_{t}^{i, N}$ cannot be computed exactly. The algorithm involves their approximation by a discrete-time stochastic process $\left(Y_{k \Delta t}^{i}, 1 \leq i \leq N\right)$, where $\Delta t$ is a discretization step of the time interval $[0, T]$. The function $V(k \Delta t, x)$ is approximated thanks to the empirical cumulative distribution function

$$
\bar{V}_{k \Delta t}(x)=\frac{1}{N} \sum_{i=1}^{N} H\left(x-Y_{k \Delta t}^{i}\right)
$$

of the numerical particle system.

Under smoothness hypotheses on $V_{0}$ and $A$, we prove that

$$
\mathbb{E}\left\|V(T, \cdot)-\bar{V}_{T}(\cdot)\right\|_{L^{1}(\mathbb{R})}+\sup _{x \in \mathbb{R}}\left(\mathbb{E}\left|V(T, x)-\bar{V}_{T}(x)\right|\right)=\mathcal{O}\left(\frac{1}{\sqrt{N}}+\Delta t\right) .
$$

The first work on the optimal rate of convergence of the Euler scheme for interacting particle systems is due to Kohatsu-Higa and Ogawa 77. They analyze the convergence of the weak approximation of a general nonlinear diffusion process of the form:

$$
\left\{\begin{array}{l}
d X_{t}=a\left(X_{t}, F * u_{t}\left(X_{t}\right)\right) d t+b\left(X_{t}, G * u_{t}\left(X_{t}\right)\right) d W_{t}, \\
\quad \text { where } u_{t} \text { is the law of } X_{t} \\
X_{t=0}=X_{0} \quad \text { with law } m_{0} .
\end{array}\right.
$$

Assuming that the functions $a, b, F$ and $G$ are smooth with bounded derivatives, they use Malliavin calculus to show that, for any $C^{\infty}$ function $f$ whose derivatives have polynomial growth at infinity,

$$
\mathbb{E}\left|\frac{1}{N} \sum_{i=1}^{N} f\left(\bar{X}_{k \Delta t}^{i}\right)-\mathbb{E} f\left(X_{t_{k}}\right)\right| \leq C\left(\frac{1}{\sqrt{N}}+\Delta t\right),
$$

where $C$ is independent of $\Delta t$ and $N$ but depends on $f$ and $\left(\bar{X}_{k \Delta t}^{i}\right)_{i=1, \ldots, N}$ is the corresponding discrete time system of interacting particles.

In the context of the present paper, $a$ is $A^{\prime}$, the diffusion coefficient $b$ is a constant and $F$ is the bounded but discontinuous Heaviside function $H$. Furthermore, we approximate the cumulative distribution function of $X_{t}$. The main difficulty in our analysis of the rate of convergence is the discontinuity of the kernel $H$. We do not use Malliavin calculus, but we take advantage of the constant diffusion coefficient to adapt some techniques developed by Talay and Tubaro [9] in their study of the global error of the Euler scheme for stochastic differential equations that are linear in the sense of McKean.

We should mention that the algorithm and its rate of convergence result could be extended to a larger class of initial data by considering $V_{0}$ as the distribution function of a signed and finite measure. Instead of identical weights equal to $1 / N$, the particles should have signed weights, fixed at time 0 and chosen according to the signed initial measure $m_{0}$. See [6] for the probabilistic interpretation of (1.1) in this particular case and [3] for a description of the algorithm using signed weights for the Burgers equation (1.2).

In Section 2, we describe the algorithm and state our main result. Section 3 is devoted to the proof of the rate of convergence. In Section 4, we conclude by giving some numerical experiments using a Romberg extrapolation procedure between 
approximation values produced by the Euler scheme to speed up the convergence with respect to the time step. Our analysis of the convergence, based on the weak convergence of the Euler scheme, lets us expect that an expansion of the error up to the order two in term of $\Delta t$ may be proved, which will justify the Romberg extrapolation.

\section{Algorithm And COnVERgence Rate}

Let us state our hypotheses.

(H1) The function $A$ is of class $C^{3}$ and $\sigma>0$.

(H2) There exists a probability measure $m_{0}$ on $\mathbb{R}$ such that the initial condition of (1.1) is given by

$$
V_{0}(x)=H * m_{0}(x)
$$

(H3) (i) The measure $m_{0}$ is absolutely continuous with respect to the Lebesgue measure. Its density $U_{0}$ is a bounded function with a bounded first order derivative.

(ii) Moreover, there exist constants $M>0, \eta \geq 0$ and $\alpha>0$ such that $\left|U_{0}\right|(x) \leq \eta \exp \left(-\alpha x^{2} / 2\right)$, when $|x|>M$.

Hypotheses (H2) and (H3) both concern the initial data $V_{0}$. (H2) restricts the framework of the algorithm presented below to a particle method with identical weights $1 / N$. It could be extend to $V_{0}(x)=\beta+H * m_{0}(x)$, where $m_{0} \neq 0$ is a signed and bounded measure and $\beta$ is a constant, using signed and weighted particles. (H3)(i) states that $V_{0}(x)$ is in $C_{b}^{2}(\mathbb{R})$ and implies, combined with (H1), that the weak solution $V(t, x)$ of (1.1) given in Proposition 1.2 is the classical one. More precisely, $V(t, x)$ is a bounded function in $C^{1,2}([0, T] \times \mathbb{R})\left(C^{1}\right.$ in the time variable $t$ and $C^{2}$ in the space variable $\left.x\right)$, with bounded first order derivatives in $t$ and $x$ and a bounded second order derivative in $x$ (see Remark [3.7). (H3)(ii), which controls the decay at infinity of the first order derivative of $V_{0}$, allows us to upper-bound the $L^{1}(\mathbb{R})$-norm of the error at time 0 . The exponential decay assumed with (H3)(ii) permits us to conclude easily (see Lemma 2.1).

We construct a family $\left(y_{0}^{i}\right)_{1 \leq i \leq N}$ of initial positions such that the piecewise constant function

$$
\bar{V}_{0}(x)=\frac{1}{N} \sum_{i=1}^{N} H\left(x-y_{0}^{i}\right)
$$

approximates $V_{0}(x)=H * U_{0}(x)$. For example, we can choose deterministic positions by inverting the function $V_{0}(x)$ :

$$
y_{0}^{i}=\left\{\begin{array}{l}
\inf \left\{y ; \int_{-\infty}^{y} U_{0}(x) d x=\frac{i}{N}\right\}, \quad i=1, \ldots, N-1, \\
\inf \left\{y ; \int_{-\infty}^{y} U_{0}(x) d x=1-\frac{1}{2 N}\right\}, \quad i=N .
\end{array}\right.
$$

By construction,

$$
\left\|V_{0}-\bar{V}_{0}\right\|_{L^{\infty}(\mathbb{R})} \leq 1 / N
$$

and the convergence for the $L^{1}(\mathbb{R})$-norm is described by 
Lemma 2.1. (Bossy and Talay [3]). Assume (H3). Then, there exists a constant $C$ depending on $U_{0}$ such that

$$
\left\|V_{0}-\bar{V}_{0}\right\|_{L^{1}(\mathbb{R})} \leq C \sqrt{\log (N)} / N .
$$

If the density $U_{0}$ has a compact support, the bound is $C / N$.

Let $\bar{m}_{0}$ denote the associated empirical measure

$$
\bar{m}_{0}=\frac{1}{N} \sum_{i=1}^{N} \delta_{y_{0}^{i}} .
$$

With $N$ fixed, on a filtered probability space $\left(\Omega, \mathcal{F}, \mathbb{P},\left(\mathcal{F}_{t}\right)_{t \geq 0}\right)$, we consider an $N$-dimensional $\left(\mathcal{F}_{t}\right)$-Brownian motion $\left(W^{1}, \ldots, W^{N}\right)$. As suggested by the propagation of chaos result (Proposition 1.2), to construct an approximation of $V(t, x)$, we have to move the $N$ particles according to the following system of stochastic differential equations

$$
\left\{\begin{array}{l}
d X_{t}^{i}=\sigma d W_{t}^{i}+A^{\prime}\left(\frac{1}{N} \sum_{j=1}^{N} H\left(X_{t}^{i}-X_{t}^{j}\right)\right) d t \\
X_{0}^{i}=y_{0}^{i}, \quad i=1, \ldots, N
\end{array}\right.
$$

The piecewise constant function

$$
\widehat{V}(t, x)=\frac{1}{N} \sum_{i=1}^{N} H\left(x-X_{t}^{i}\right)
$$

approximates $V(t, x)$ with an error depending on $N$ only. To get a simulation procedure for a trajectory of each $\left(X^{i}\right)$, we discretize in time. We choose $\Delta t$ and $K \in \mathbb{N}$ such that $T=\Delta t K$ and denote by $t_{k}=k \Delta t$ the discrete times, with $1 \leq k \leq K$. The Euler scheme leads to the following discrete-time system

$$
\left\{\begin{array}{l}
Y_{t_{k+1}}^{i}=Y_{t_{k}}^{i}+\sigma\left(W_{t_{k+1}}^{i}-W_{t_{k}}^{i}\right)+\Delta t A^{\prime}\left(\frac{1}{N} \sum_{j=1}^{N} H\left(Y_{t_{k}}^{i}-Y_{t_{k}}^{j}\right)\right), \\
Y_{0}^{i}=y_{0}^{i}, \quad i=1, \ldots, N .
\end{array}\right.
$$

We approximate $V\left(t_{k}, x\right)$, the solution of (1.1), by the piecewise constant function

$$
\bar{V}_{t_{k}}(x)=\frac{1}{N} \sum_{i=1}^{N} H\left(x-Y_{t_{k}}^{i}\right) \text {. }
$$

The estimate on the convergence rate is

Theorem 2.2. Assume (H1), (H2) and (H3). For $T>0$ fixed, let $\Delta t>0$ be such that $T=\Delta t K, K \in \mathbb{N}$. Let $V\left(t_{k}, x\right)$ be the solution at time $t_{k}=k \Delta t$ of (1.1) with initial condition $V_{0}$. Let $\bar{V}_{t_{k}}(x)$ be defined as in (2.4) with $N$ particles. Then there exists a positive constant $C$, depending only on $V_{0}, A, \sigma$ and $T$, such that for all $k$ in $\{1, \ldots, K\}$,

$$
\sup _{x \in \mathbb{R}} \mathbb{E}\left|V\left(t_{k}, x\right)-\bar{V}_{t_{k}}(x)\right| \leq C\left(\left\|V_{0}-\bar{V}_{0}\right\|_{L^{\infty}(\mathbb{R})}+\frac{1}{\sqrt{N}}+\Delta t\right)
$$

and

$$
\mathbb{E}\left\|V\left(t_{k}, \cdot\right)-\bar{V}_{t_{k}}(\cdot)\right\|_{L^{1}(\mathbb{R})} \leq C\left(\left\|V_{0}-\bar{V}_{0}\right\|_{L^{1}(\mathbb{R})}+\frac{1}{\sqrt{N}}+\Delta t\right) .
$$




\section{Proof of Theorem 2.2}

In the sequel, we will use the continuous version of the discrete time processes $\left(Y^{i}\right)$, which consists in freezing the drift coefficient on each interval $\left[t_{k}, t_{k+1}\right]$ :

$$
Y_{t}^{i}=y_{0}^{i}+\int_{0}^{t} A^{\prime}\left(\bar{V}_{\eta(s)}\left(Y_{\eta(s)}^{i}\right)\right) d s+\sigma W_{t}^{i},
$$

where $\eta(s)=\sup _{k \in[0, \ldots, K]}\left\{t_{k} ; t_{k} \leq s\right\}$. Also $C$ denotes any positive constant depending only on $T, \sigma, A$ and $V_{0}$; for any strictly positive constant $\alpha, g_{\alpha}$ denotes the Gaussian density function

$$
g_{\alpha}(x)=\frac{1}{\sqrt{2 \pi \alpha}} \exp \left(-\frac{x^{2}}{2 \alpha}\right) .
$$

According to the probabilistic interpretation given in Section 1, the solution of (1.1) is given by

$$
V(t, x)=H * P_{t}(x)=\mathbb{E}_{P}\left(H\left(x-X_{t}\right)\right),
$$

where $P$ is the solution of the martingale problem (1.3) and $X$ denotes the canonical process on $C([0,+\infty), \mathbb{R})$. We define the real valued function $B(t, x)$ by

$$
B(t, x)=A^{\prime}(V(t, x)), \quad(t, x) \in[0, T] \times \mathbb{R}
$$

and consider the Markov process $(Z)$ solution of

$$
\left\{\begin{array}{l}
Z_{t}=Z_{0}+\int_{0}^{t} B\left(s, Z_{s}\right) d s+\sigma W_{t}, \quad t \in[0, T], \\
Z_{0} \quad \text { with law } m_{0}
\end{array}\right.
$$

where $(W)$ is a one-dimensional Brownian motion independent of $\left(W^{1}, \ldots, W^{N}\right)$. By Proposition 1.2, the law of $(Z)$ solves the martingale problem (1.3) and thus $V(t, x)=\mathbb{E} H\left(x-Z_{t}\right)$.

Let $\left(Z^{0, y}\right)$ be the solution of the stochastic differential equation (3.3) with the deterministic initial condition $y$ at time 0 (i.e., $Z_{0}=y$ ). More generally, for any $0 \leq s \leq T,\left(Z_{t}^{s, y}, t \in[s, T]\right)$ denotes the solution of

$$
Z_{t}^{s, y}=y+\int_{s}^{t} B\left(\theta, Z_{\theta}^{s, y}\right) d \theta+\sigma\left(W_{t}-W_{s}\right), \quad t \in[s, T] .
$$

We will prove below that the drift function $B(t, x)$ is smooth, so that the strong existence and uniqueness of this solution are ensured.

Let $k$ be in $\{1, \ldots, K\}$. To prove Theorem 2.2, we start from

$$
V\left(t_{k}, x\right)-\bar{V}_{t_{k}}(x)=\mathbb{E} H\left(x-Z_{t_{k}}\right)-\frac{1}{N} \sum_{i=1}^{N} H\left(x-Y_{t_{k}}^{i}\right) .
$$

First, we introduce an artificial smoothing of the Heaviside function. For an arbitrary constant $\varepsilon>0$, we define the function $H_{\varepsilon}(x)=g_{\varepsilon} * H(x)$ and we decompose 
the expression above into four parts:

$$
\begin{aligned}
V\left(t_{k}, x\right)-\bar{V}_{t_{k}}(x)= & \mathbb{E} H\left(x-Z_{t_{k}}\right)-\mathbb{E} H_{\varepsilon}\left(x-Z_{t_{k}}\right) \\
& +\int_{\mathbb{R}} \mathbb{E} H_{\varepsilon}\left(x-Z_{t_{k}}^{0, y}\right) m_{0}(d y)-\int_{\mathbb{R}} \mathbb{E} H_{\varepsilon}\left(x-Z_{t_{k}}^{0, y}\right) \bar{m}_{0}(d y) \\
& +\frac{1}{N} \sum_{i=1}^{N}\left[\mathbb{E} H_{\varepsilon}\left(x-Z_{t_{k}}^{0, y_{0}^{i}}\right)-H_{\varepsilon}\left(x-Y_{t_{k}}^{i}\right)\right] \\
& +\frac{1}{N} \sum_{i=1}^{N}\left[H_{\varepsilon}\left(x-Y_{t_{k}}^{i}\right)-H\left(x-Y_{t_{k}}^{i}\right)\right] .
\end{aligned}
$$

The first and last terms are smoothing errors and will tend to zero with $\varepsilon$. The second term corresponds to the propagation at time $t_{k}$ of the initialization error $\left|V_{0}(x)-\bar{V}_{0}(x)\right|$.

To let the reader understand the third term, we transform it: for any time $t_{k}$ and any $x \in \mathbb{R}$, we consider the partial differential equation

$$
\left\{\begin{array}{c}
\frac{\partial v_{t_{k}, x}}{\partial s}(s, y)+\frac{1}{2} \sigma^{2} \frac{\partial^{2} v_{t_{k}, x}}{\partial y^{2}}(s, y)+B(s, y) \frac{\partial v_{t_{k}, x}}{\partial y}(s, y)=0 \\
\forall(s, y) \in\left[0, t_{k}\right) \times \mathbb{R} \\
v_{t_{k}, x}\left(t_{k}, y\right)=H_{\varepsilon}(x-y), \forall y \in \mathbb{R} .
\end{array}\right.
$$

From Lemma 3.9 below, 3.6 has a unique bounded classical solution $v_{t_{k}, x}(s, y)$ that is a bounded function in $C^{1,2}\left(\left[0, t_{k}\right) \times \mathbb{R}\right)$. Hence, by the Feynman-Kac representation of a Cauchy problem, $v_{t_{k}, x}(s, y)=\mathbb{E} H_{\varepsilon}\left(x-Z_{t_{k}}^{s, y}\right)$ and

$$
\begin{aligned}
\mathbb{E} H_{\varepsilon}\left(x-Z_{t_{k}}^{0, y_{0}^{i}}\right)-H_{\varepsilon}\left(x-Y_{t_{k}}^{i}\right) & =v_{t_{k}, x}\left(0, y_{0}^{i}\right)-v_{t_{k}, x}\left(t_{k}, Y_{t_{k}}^{i}\right) \\
& =\sum_{l=0}^{k-1}\left(v_{t_{k}, x}\left(t_{l}, Y_{t_{l}}^{i}\right)-v_{t_{k}, x}\left(t_{l+1}, Y_{t_{l+1}}^{i}\right)\right) .
\end{aligned}
$$

As $v_{t_{k}, x}$ is solution of (3.6), the Itô formula gives

$$
\begin{aligned}
\frac{1}{N} \sum_{i=1}^{N} & \left(\mathbb{E} H_{\varepsilon}\left(x-Z_{t_{k}}^{0, y_{0}^{i}}\right)-H_{\varepsilon}\left(x-Y_{t_{k}}^{i}\right)\right) \\
= & \frac{1}{N} \sum_{i=1}^{N} \sum_{l=0}^{k-1} \int_{t_{l}}^{t_{l+1}} \frac{\partial v_{t_{k}, x}}{\partial y}\left(s, Y_{s}^{i}\right)\left(B\left(s, Y_{s}^{i}\right)-A^{\prime}\left(\bar{V}_{t_{l}}\left(Y_{t_{l}}^{i}\right)\right)\right) d s \\
& -\frac{1}{N} \sum_{i=1}^{N} \int_{0}^{t_{k}} \sigma \frac{\partial v_{t_{k}, x}}{\partial y}\left(s, Y_{s}^{i}\right) d W_{s}^{i} .
\end{aligned}
$$

The second term of the right-hand side of (3.7) is a statistical error. We will bound the expectation of its absolute value by $C / \sqrt{N}$. The first term in the right-hand side of (3.7) is the discretization error where the most important difficulties of the proof are concentrated.

In the next subsections, we give the proof of these four lemmas:

Lemma 3.1. Smoothing error. For any $x$ in $\mathbb{R}, \int_{\mathbb{R}}\left|H(x-z)-H_{\varepsilon}(x-z)\right| d z \leq C \sqrt{\varepsilon}$. Assume (H1), (H2) and (H3). Then,

$$
\sup _{x \in \mathbb{R}}\left|\mathbb{E} H\left(x-Z_{t_{k}}\right)-\mathbb{E} H_{\varepsilon}\left(x-Z_{t_{k}}\right)\right| \leq C \sqrt{\varepsilon}
$$


and for any $i$ and $j$ in $\{1, \ldots, N\}$, with $j \neq i$, and any $k$ in $\{1, \ldots, K\}$,

$$
\begin{aligned}
& \sup _{x \in \mathbb{R}} \mathbb{E}\left|H_{\varepsilon}\left(x-Y_{t_{k}}^{i}\right)-H\left(x-Y_{t_{k}}^{i}\right)\right| \leq C \sqrt{\varepsilon} / \sqrt{\Delta t}, \\
& \mathbb{E}\left|H_{\varepsilon}\left(Y_{t_{k}}^{j}-Y_{t_{k}}^{i}\right)-H\left(Y_{t_{k}}^{j}-Y_{t_{k}}^{i}\right)\right| \leq C \sqrt{\varepsilon} / \sqrt{\Delta t} .
\end{aligned}
$$

The positive constant $C$ depends on $V_{0}, A, \sigma$ and $T$ only.

Lemma 3.2. Initialization error. Assume (H1), (H2) and (H3). For all $k$ in $\{1, \ldots, K\}$,

$$
\begin{aligned}
& \sup _{x \in \mathbb{R}}\left|\int_{\mathbb{R}} \mathbb{E} H_{\varepsilon}\left(x-Z_{t_{k}}^{0, y}\right) m_{0}(d y)-\int_{\mathbb{R}} \mathbb{E} H_{\varepsilon}\left(x-Z_{t_{k}}^{0, y}\right) \bar{m}_{0}(d y)\right| \\
& \quad \leq C\left\|V_{0}-\bar{V}_{0}\right\|_{L^{\infty}(\mathbb{R})}
\end{aligned}
$$

and

$$
\begin{aligned}
& \left\|\int_{\mathbb{R}} \mathbb{E} H_{\varepsilon}\left(\cdot-Z_{t_{k}}^{0, y}\right) m_{0}(d y)-\int_{\mathbb{R}} \mathbb{E} H_{\varepsilon}\left(\cdot-Z_{t_{k}}^{0, y}\right) \bar{m}_{0}(d y)\right\|_{L^{1}(\mathbb{R})} \\
& \quad \leq C\left\|V_{0}-\bar{V}_{0}\right\|_{L^{1}(\mathbb{R})} .
\end{aligned}
$$

The positive constant $C$ depends on $V_{0}, A, \sigma$ and $T$ only.

Lemma 3.3. Statistical error. Assume (H1), (H2) and (H3). For all $k$ in $\{1, \ldots, K\}$,

$$
\sup _{x \in \mathbb{R}} \mathbb{E}\left|\frac{1}{N} \sum_{i=1}^{N} \int_{0}^{t_{k}} \sigma \frac{\partial v_{t_{k}, x}}{\partial y}\left(s, Y_{s}^{i}\right) d W_{s}^{i}\right| \leq \frac{C}{\sqrt{N}}
$$

and

$$
\mathbb{E}\left\|\frac{1}{N} \sum_{i=1}^{N} \int_{0}^{t_{k}} \sigma \frac{\partial v_{t_{k}, \cdot}}{\partial y}\left(s, Y_{s}^{i}\right) d W_{s}^{i}\right\|_{L^{1}(\mathbb{R})} \leq \frac{C}{\sqrt{N}} .
$$

The positive constant $C$ depends on $V_{0}, A, \sigma$ and $T$ only.

Lemma 3.4. Discretization error. Assume (H1), (H2) and (H3). For all $k$ in $\{1, \ldots, K\}$,

$$
\begin{aligned}
\sup _{x \in \mathbb{R}} \mathbb{E} & \left|\frac{1}{N} \sum_{i=1}^{N} \sum_{l=0}^{k-1} \int_{t_{l}}^{t_{l+1}} \frac{\partial v_{t_{k}, x}}{\partial y}\left(s, Y_{s}^{i}\right)\left(B\left(s, Y_{s}^{i}\right)-A^{\prime}\left(\bar{V}_{t_{l}}\left(Y_{t_{l}}^{i}\right)\right)\right) d s\right| \\
\leq & \leq\left(\frac{1}{\sqrt{N}}+\Delta t\right)
\end{aligned}
$$

and

$$
\begin{aligned}
& \mathbb{E}\left\|\frac{1}{N} \sum_{i=1}^{N} \sum_{l=0}^{k-1} \int_{t_{l}}^{t_{l+1}} \frac{\partial v_{t_{k}, \cdot}}{\partial y}\left(s, Y_{s}^{i}\right)\left(B\left(s, Y_{s}^{i}\right)-A^{\prime}\left(\bar{V}_{t_{l}}\left(Y_{t_{l}}^{i}\right)\right)\right) d s\right\|_{L^{1}(\mathbb{R})} \\
& \quad \leq C\left(\frac{1}{\sqrt{N}}+\Delta t\right) .
\end{aligned}
$$

The positive constant $C$ depends on $V_{0}, A, \sigma$ and $T$ only. 
We choose $\varepsilon=\Delta t^{3}$. Estimates of the four above lemmas combined with equalities (3.7) and (3.5) prove Theorem 2.2.

The section is organized as follows. In Subsection 3.1 we prove some preliminary estimates and regularity results on the drift function $B$ and on the solution $v_{t_{k}, x}$ of equation (3.6). Then, we successively prove Lemmas 3.1 3.2 and 3.3. We finish by the proof of the main Lemma 3.4

3.1. Preliminary lemmas. Consider the process $(Z)$ solution of 3.3 . The drift function $B$ defined in (3.2) is bounded by $\sup _{[0,1]}\left|A^{\prime}(v)\right|$. Hence, by the Girsanov theorem, for any $t>0, Z_{t}$ has a density denoted by $U(t, \cdot)$. Furthermore,

Remark 3.5. The transition probability $\mathbb{P}\left(t, d z ; s, Z_{s}=y\right)$ has a density that we denote by $\Gamma(t, z ; s, y)$, which is in $L^{2}(\mathbb{R})$. Moreover, for all $y \in \mathbb{R}$,

$$
\|\Gamma(t, \cdot ; s, y)\|_{L^{2}(\mathbb{R})} \leq \frac{C}{(t-s)^{1 / 4}},
$$

where the positive constant $C$ depends on $\sigma, T$ and $A$ only and, therefore, is uniform in $y$. This can be proven by using the Girsanov theorem (see the proof of Proposition 1.1 in 8]). In particular, $U(t, \cdot)$ is in $L^{2}(\mathbb{R})$ for all $t>0$ and, without any hypothesis on $m_{0}$,

$$
\|U(t, \cdot)\|_{L^{2}(\mathbb{R})} \leq \frac{C}{t^{1 / 4}} .
$$

Lemma 3.6. Assume (H1), (H2) and (H3). The density $U(t, x)$ of $Z_{t}$ is bounded uniformly in $t \in[0, T]$ and has a first partial derivative in $x$ which is bounded uniformly in $t \in[0, T]$. The function $B(t, x)$ is in $C^{1,2}([0, T] \times \mathbb{R})$ and its derivatives $\frac{\partial B}{\partial t}(t, x), \frac{\partial B}{\partial x}(t, x)$ and $\frac{\partial^{2} B}{\partial x^{2}}(t, x)$ are bounded uniformly in $t \in[0, T]$.

Remark 3.7. Even if this is not explicitly stated in Lemma 3.6, one can easily deduce from the following proof that $V$ is in $C^{1,2}([0, T] \times \mathbb{R})$ with bounded first order derivatives in the time and space variables and bounded second order derivative in the space variable. Thus, $V$ is the bounded classical solution of the scalar conservation law (1.1).

Proof. For all $t>0, g_{\sigma^{2} t}(x)$ denotes the density of the Gaussian random variable $\sigma W_{t}$. Let $S_{t}$ be the corresponding semi-group defined by $S_{t} f=g_{\sigma^{2} t} * f$. Let us show that $U$ is the unique weak solution in $L^{1}(\mathbb{R})$ of the following integral linear Fokker Planck equation

$$
\left.\left.p_{t}=S_{t} U_{0}-\int_{0}^{t} \frac{\partial}{\partial x} S_{t-s}\left(B(s, \cdot) p_{s}\right) d s, \forall t \in\right] 0, T\right], p_{0}=U_{0},
$$

where $U_{0}$ is the density of $m_{0}$. We will deduce from (3.10) the regularity results of the lemma. For a fixed $t$ in $(0, T]$ and a function $f$ in $C^{\infty}(\mathbb{R})$ with compact support, we set $G(s, x)=S_{t-s} f(x)$, for all $s \in[0, t)$. Then, $G$ is the solution of the backward heat equation

$$
\left\{\begin{array}{l}
\frac{\partial G}{\partial s}+\frac{1}{2} \sigma^{2} \frac{\partial^{2} G}{\partial x^{2}}=0, \quad 0 \leq s<t \\
G(t, x)=f(x)
\end{array}\right.
$$

By applying Itô's formula to $G\left(t, Z_{t}\right)$ and taking the expectation, we obtain that

$$
\int_{\mathbb{R}} f(x) U(t, x) d x=\int_{\mathbb{R}} G(0, x) U_{0}(x) d x+\int_{0}^{t} \int_{\mathbb{R}} \frac{\partial G}{\partial x}(s, x) B(s, x) U(s, x) d x
$$


and the definition of $G(s, x)$ leads to

$$
\begin{aligned}
\int_{\mathbb{R}} f(x) U(t, x) d x= & \int_{\mathbb{R}} S_{t} f(x) U_{0}(x) d x \\
& +\int_{0}^{t} \int_{\mathbb{R}}\left(\int_{\mathbb{R}} g_{\sigma^{2}(t-s)}^{\prime}(x-y) f(y) d y\right) B(s, x) U(s, x) d x d s .
\end{aligned}
$$

Moreover,

$$
\begin{aligned}
\int_{0}^{t} \int_{\mathbb{R}} & \left(\int_{\mathbb{R}} g_{\sigma^{2}(t-s)}^{\prime}(x-y) f(y) d y\right) B(s, x) U(s, x) d x d s \\
= & -\int_{0}^{t} \int_{\mathbb{R}} f(y) \frac{\partial}{\partial y}\left(\int_{\mathbb{R}} g_{\sigma^{2}(t-s)}(x-y) B(s, x) U(s, x) d x\right) d y d s \\
& =-\int_{0}^{t} \int_{\mathbb{R}} f(y) \frac{\partial}{\partial y} S_{t-s}(B(s, \cdot) U(s, \cdot))(y) d y d s .
\end{aligned}
$$

Hence,

$$
\begin{aligned}
\int_{\mathbb{R}} f(x) U(t, x) d x= & \int_{\mathbb{R}} f(x) S_{t} U_{0}(x) d x \\
& -\int_{0}^{t} \int_{\mathbb{R}} f(x) \frac{\partial}{\partial x} S_{t-s}(B(s, \cdot) U(s, \cdot))(x) d x d s
\end{aligned}
$$

which means that $U$ satisfies $(3.10)$ in the weak sense. Now, consider two solutions $p^{1}$ and $p^{2}$ in $L^{1}(\mathbb{R})$ of (3.10). For all $t \in(0, T]$,

$$
\begin{aligned}
\left\|p_{t}^{1}-p_{t}^{2}\right\|_{L^{1}(\mathbb{R})} & =\left\|\int_{0}^{t} \frac{\partial}{\partial x} S_{t-s}\left(B(s, \cdot)\left(p_{s}^{1}-p_{s}^{2}\right)\right) d s\right\|_{L^{1}(\mathbb{R})} \\
& \leq \sup _{u \in[0,1]}\left|A^{\prime}(u)\right| \int_{0}^{t}\left\|g_{\sigma^{2}(t-s)}^{\prime}\right\|_{L^{1}(\mathbb{R})}\left\|p_{s}^{1}-p_{s}^{2}\right\|_{L^{1}(\mathbb{R})} d s \\
& \leq \int_{0}^{t} \frac{C}{\sqrt{t-s}}\left\|p_{s}^{1}-p_{s}^{2}\right\|_{L^{1}(\mathbb{R})} d s .
\end{aligned}
$$

We conclude on the uniqueness of the solution of (3.10) by applying Gronwall's lemma. We have now that for all $t \in(0, T]$ and $x \in \mathbb{R}$,

$$
U(t, x)=g_{\sigma^{2} t} * U_{0}(x)-\int_{0}^{t} g_{\sigma^{2}(t-s)}^{\prime} *(B(s, \cdot) U(s, \cdot))(x) d s .
$$

Let us prove that $U$ is bounded uniformly in $t \in[0, T]$.

$$
\begin{aligned}
U(t, x) & \leq\left\|U_{0}\right\|_{L^{\infty}(\mathbb{R})}+\sup _{[0,1]}\left|A^{\prime}\right| \int_{0}^{t} \int_{\mathbb{R}}\left|g_{\sigma^{2}(t-s)}^{\prime}\right|(x-y) U(s, y) d y d s \\
& \leq\left\|U_{0}\right\|_{L^{\infty}(\mathbb{R})}+\int_{0}^{t} \frac{C}{\sqrt{(t-s)}} \sqrt{\int_{\mathbb{R}} g_{2 \sigma^{2}(t-s)}(x-y) U^{2}(s, y) d y} d s \\
& \leq\left\|U_{0}\right\|_{L^{\infty}(\mathbb{R})}+\int_{0}^{t} \frac{C}{(t-s)^{3 / 4} s^{1 / 4}} d s .
\end{aligned}
$$

The last upper bound above is obtained by Remark 3.5. Thus, $\|U\|_{L^{\infty}([0, T] \times \mathbb{R})} \leq C$, where the constant $C$ depends on $\sigma, T, A$ and $U_{0}$ only. Now we remark that $\frac{\partial B}{\partial x}(t, \cdot)=A^{\prime \prime}\left(\mathbb{E} H\left(x-Z_{t}\right)\right) U(t, x)$, and hence, $\left\|\frac{\partial B}{\partial x}\right\|_{L^{\infty}([0, T] \times \mathbb{R})} \leq C$. 
If we formally derive (3.11), we obtain that $\frac{\partial U}{\partial x}$ must satisfy the equation

$$
\begin{aligned}
\frac{\partial U}{\partial x}(t, x)= & g_{\sigma^{2} t} * U_{0}^{\prime}(x) \\
& -\int_{0}^{t} g_{\sigma^{2}(t-s)}^{\prime} *\left(\frac{\partial B}{\partial x}(s, \cdot) U(s, \cdot)+B(s \cdot \cdot) \frac{\partial U}{\partial x}(s, \cdot)\right)(x) d s .
\end{aligned}
$$

Let us prove that $\frac{\partial U}{\partial x}$ satisfies 3.12 and more precisely that $\frac{\partial U}{\partial x}$ is in $C([0, T]$, $L^{1}(\mathbb{R}) \cap C_{b}(\mathbb{R})$ ), where $C_{b}(\mathbb{R})$ denotes the set of bounded continuous functions on $\mathbb{R}$. Let $E_{[0, T]}$ be the space

$$
E_{[0, T]}=\left\{u \in C\left([0, T], L^{1}(\mathbb{R}) \cap C_{b}(\mathbb{R})\right),\|u\|_{E_{[0, T]}}=\sup _{t \in[0, T]}\|u(t)\|_{E}<+\infty\right\},
$$

with $\|f\|_{E}=\|f\|_{L^{1}(\mathbb{R})}+\sup _{x \in \mathbb{R}}|f(x)|+\left\|\int_{-\infty}^{.} f(y) d y\right\|_{L^{1}(\mathbb{R})}$. Let $\Upsilon: E_{[0, T]} \longrightarrow E_{[0, T]}$ be defined by

$$
\begin{aligned}
\Upsilon(u)(t, x)= & g_{\sigma^{2} t} * U_{0}^{\prime}(x) \\
& -\int_{0}^{t} g_{\sigma^{2}(t-s)}^{\prime} *\left(\frac{\partial B}{\partial x}(s, \cdot)\left(\int_{-\infty}^{\cdot} u(s, y) d y\right)+B(s, \cdot) u(s, \cdot)\right)(x) d s .
\end{aligned}
$$

We will show that $\frac{\partial U}{\partial x}$ is the fixed point in $E_{[0, T]}$ of the application $\Upsilon$. For $u^{1}$ and $u^{2}$ in $E_{[0, T]}$,

$$
\begin{aligned}
& \left(\Upsilon\left(u^{1}\right)-\Upsilon\left(u^{2}\right)\right)(t, x) \\
& =\int_{0}^{t} g_{\sigma^{2}(t-s)}^{\prime} *\left(\frac{\partial B}{\partial x}(s, \cdot)\left(\int_{-\infty}^{\cdot}\left(u^{1}-u^{2}\right)(s, y) d y\right)+B(s, \cdot)\left(u^{1}-u^{2}\right)(s, \cdot)\right)(x) d s .
\end{aligned}
$$

An easy computation shows that

$$
\begin{aligned}
& \left\|\left(\Upsilon\left(u^{1}\right)-\Upsilon\left(u^{2}\right)\right)(t)\right\|_{E} \\
& \quad \leq \int_{0}^{t}\left\|g_{\sigma^{2}(t-s)}^{\prime}\right\|_{L^{1}(\mathbb{R})}\left\||B|+\left|\frac{\partial B}{\partial x}\right|\right\|_{L^{\infty}([0, T] \times \mathbb{R})}\left\|\left(u^{1}-u^{2}\right)(s)\right\|_{E} d s .
\end{aligned}
$$

Let $t_{0}$ such that $\int_{0}^{t_{0}} \frac{2 D}{\sqrt{2 \pi \sigma^{2}(t-s)}} d s=\frac{1}{2}$, where $D=\left\||B|+\left|\frac{\partial B}{\partial x}\right|\right\|_{L^{\infty}([0, T] \times \mathbb{R})}$. We deduce from the previous inequality that $\Upsilon$ is a contraction on $E_{\left[0, t_{0}\right]}$ and we denote $\nu$ its fixed point. For any $u \in E_{[0, T]}$ and $t \in\left(t_{0}, T\right]$, we remark that

$$
\begin{aligned}
\Upsilon(u)(t, x)= & g_{\sigma^{2}\left(t-t_{0}\right)} * \Upsilon\left(u\left(t_{0}\right)\right)(x) \\
& -\int_{t_{0}}^{t} g_{\sigma^{2}(t-s)}^{\prime} *\left(\frac{\partial B}{\partial x}(s, \cdot)\left(\int_{-\infty}^{.} u(s, y) d y\right)+B(s, \cdot) u(s, \cdot)\right)(x) d s .
\end{aligned}
$$

If $\nu^{1}$ and $\nu^{2}$ in $E_{\left[0,2 t_{0}\right]}$ are such that $\nu^{1}(t)=\nu^{2}(t)=\nu(t)$ for $t \in\left[0, t_{0}\right]$, from the expression above we easily get that

$$
\begin{aligned}
& \left\|\left(\Upsilon\left(\nu^{1}\right)-\Upsilon\left(\nu^{2}\right)\right)(t)\right\|_{E} \\
& \quad \leq \int_{t_{0}}^{2 t_{0}}\left\|g_{\sigma^{2}(t-s)}^{\prime}\right\|_{L^{1}(\mathbb{R})}\left\||B(s, \cdot)|+\left|\frac{\partial B}{\partial x}(s, \cdot)\right|\right\|_{L^{\infty}(\mathbb{R})}\left\|\left(\nu^{1}-\nu^{2}\right)(s)\right\|_{E} d s
\end{aligned}
$$

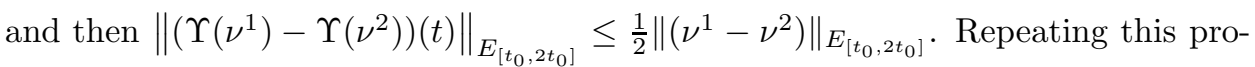
cedure, we construct the fixed point $\nu$ of $\Upsilon$ on $E_{[0, T]}$. Now, we remark that the 
function $(t, x) \longrightarrow \int_{-\infty}^{x} \nu(t, y) d y$ is the solution in $L^{1}(\mathbb{R})$ of (3.10). By the uniqueness of the solution of (3.10), $U(t, x)=\int_{-\infty}^{x} \nu(t, y) d y$ and $\frac{\partial U}{\partial x}(t, x)=\nu(t, x)$. From (3.12),

$$
\begin{aligned}
& \left\|\frac{\partial U}{\partial x}(t, \cdot)\right\|_{L^{\infty}(\mathbb{R})} \\
& \quad \leq\left\|U_{0}^{\prime}\right\|_{L^{\infty}(\mathbb{R})}+\frac{\sqrt{T} D}{\sqrt{2 \pi \sigma^{2}}}\|U\|_{L^{\infty}([0, T] \times \mathbb{R})}+\int_{0}^{t} \frac{2 D}{\sqrt{2 \pi \sigma^{2}(t-s)}}\left\|\frac{\partial U}{\partial x}(s, \cdot)\right\|_{L^{\infty}(\mathbb{R})} d s .
\end{aligned}
$$

We conclude that $\frac{\partial U}{\partial x}$ is bounded uniformly in $t \in[0, T]$ by Gronwall's lemma. Moreover, for all $(t, x) \in[0, T] \times \mathbb{R}$,

$$
\frac{\partial^{2} B}{\partial x^{2}}(t, x)=A^{\prime \prime \prime}\left(\mathbb{E} H\left(x-z_{t}\right)\right) U^{2}(t, x)+A^{\prime \prime}\left(\mathbb{E} H\left(x-z_{t}\right)\right) \frac{\partial U}{\partial x}(t, x)
$$

and $\left\|\frac{\partial^{2} B}{\partial x^{2}}\right\|_{L^{\infty}([0, T] \times \mathbb{R})} \leq C$. To finish the proof, we have to bound the derivative in time of the function $B$. We have

$$
\frac{\partial}{\partial t} B(t, x)=A^{\prime \prime}\left(\mathbb{E} H\left(x-Z_{t}\right)\right) \frac{\partial}{\partial t} \mathbb{E} H\left(x-Z_{t}\right)
$$

where, by (3.11),

$$
\begin{array}{rl}
\frac{\partial}{\partial t} \mathbb{E} & H\left(x-Z_{t}\right) \\
& =\frac{\partial}{\partial t} \int_{-\infty}^{x} U(t, y) d y \\
& =\frac{\sigma^{2}}{2} \frac{\partial^{2}}{\partial x^{2}} \int_{-\infty}^{x} g_{\sigma^{2} t} * U_{0}(y) d y-\frac{\partial}{\partial t} \int_{0}^{t} g_{\sigma^{2}(t-s)} *(B(s, \cdot) U(s, \cdot))(x) d s \\
& =\frac{\sigma^{2}}{2} g_{\sigma^{2} t} * U_{0}^{\prime}-B(t, x) U(t, x)-\int_{0}^{t} \frac{\sigma^{2}}{2} g_{\sigma^{2}(t-s)}^{\prime} * \frac{\partial}{\partial x}(B(s, \cdot) U(s, \cdot))(x) d s \\
& \leq\left\|U_{0}^{\prime}\right\|_{L^{\infty}(\mathbb{R})}+\|B\|_{L^{\infty}([0, T] \times \mathbb{R})}\|U\|_{L^{\infty}([0, T] \times \mathbb{R})}+\int_{0}^{t} \frac{C}{\sqrt{t-s}} d s,
\end{array}
$$

which gives that $\left\|\frac{\partial}{\partial t} B\right\|_{L^{\infty}([0, T] \times \mathbb{R})} \leq C$.

The following lemma is directly adapted from Theorem 11 in [4, Chapter 1] for our particular one-dimensional case with constant diffusion coefficient and gives exponential bound for the transition density of $Z_{t}^{s, x}$.

Lemma 3.8 (Friedman [4]). If the drift function $B(t, x)$ is a bounded continuous function on $[0, T] \times \mathbb{R}$, Hölder continuous (with exponent $\alpha<1$ ) on $\mathbb{R}$ uniformly in $t$, then the transition probability of the process $\left(Z_{t}^{s, x}\right)$ has a smooth density, denoted by $\Gamma(t, z ; s, x)$, and there exists a positive constant $C_{0}$ depending on $T, B$ and $\sigma$, such that for all $0 \leq s<t \leq T$ and $(x, z)$ in $\mathbb{R}^{2}$,

$$
\Gamma(t, z ; s, x) \leq \frac{C_{0}}{\sqrt{t-s}} \exp \left(-\frac{(x-z)^{2}}{2 \bar{\sigma}^{2}(t-s)}\right), \forall \bar{\sigma}>\sigma .
$$

In the sequel, we will choose $\bar{\sigma}=2 \sigma$. 
Lemma 3.9. Assume (H1), (H2) and (H3). The Cauchy problem (3.6) has a unique bounded solution in $C^{1,2}\left(\left[0, t_{k}\right) \times \mathbb{R}\right)$ and there exists a positive constant $C$ depending only on $A, \sigma, T$ and $V_{0}$, such that for all $(s, z)$ in $\left[0, t_{k}\right) \times \mathbb{R}$,

$$
\left|\frac{\partial v_{t_{k}, x}}{\partial z}(s, z)\right| \leq C g_{\varepsilon+2 \sigma^{2}\left(t_{k}-s\right)}(x-z) .
$$

Moreover, for all $s$ in $\left[0, t_{k}\right)$

$$
\sup _{z \in \mathbb{R}}\left\|\frac{\partial^{2} v_{t_{k}, \cdot}}{\partial z^{2}}(s, z)\right\|_{L^{1}(\mathbb{R})} \leq \frac{C}{\sqrt{t_{k}-s}}
$$

and

$$
\sup _{x \in \mathbb{R}}\left\|\left|\frac{\partial^{2} v_{t_{k}, x}}{\partial y^{2}}\right|^{5 / 4}(s, \cdot)\right\|_{L^{1}(\mathbb{R})} \leq \frac{C}{\left(t_{k}-s\right)^{3 / 4}} .
$$

Proof. Existence and uniqueness of a bounded classical solution of (3.6) can be found in Friedman [5].

By the Feynman-Kac representation, $v_{t_{k}, x}(s, y)=\mathbb{E} H_{\varepsilon}\left(x-Z_{t_{k}}^{s, y}\right)$ and $\frac{\partial v_{t_{k}, x}}{\partial y}(s, y)$ $=-\mathbb{E}\left[g_{\varepsilon}\left(x-Z_{t_{k}}^{s, y}\right) \frac{d Z_{t_{k}}^{s, y}}{d y}\right]$, with $\frac{d Z_{t_{k}}^{s, y}}{d y}=\exp \left(\int_{s}^{t_{k}} \frac{\partial B}{\partial x}\left(\theta, Z_{\theta}^{s, y}\right) d \theta\right)$. As the function $\frac{\partial B}{\partial x}(t, x)$ is bounded in $[0, T] \times \mathbb{R}$, we get

$$
\left|\frac{\partial v_{t_{k}, x}}{\partial y}(s, y)\right| \leq C\left(g_{\varepsilon} * \Gamma\left(t_{k}, \cdot ; s, y\right)\right)(x)
$$

from which, by Lemma 3.8, we deduce immediately (3.13). For the second order derivative, we have that

$$
\frac{\partial^{2} v_{t_{k}, x}}{\partial y^{2}}(s, y)=\mathbb{E}\left[g_{\varepsilon}^{\prime}\left(x-Z_{t_{k}}^{s, y}\right)\left(\frac{d Z_{t_{k}}^{s, y}}{d y}\right)^{2}-g_{\varepsilon}\left(x-Z_{t_{k}}^{s, y}\right) \frac{d^{2} Z_{t_{k}}^{s, y}}{d y^{2}}\right],
$$

with $\frac{d^{2} Z_{t_{k}}^{s, y}}{d y^{2}}=\exp \left(\int_{s}^{t_{k}} \frac{\partial B}{\partial x}\left(\theta, Z_{\theta}^{s, y}\right) d \theta\right) \int_{s}^{t_{k}} \frac{\partial^{2} B}{\partial x^{2}}\left(u, Z_{u}^{s, y}\right) \exp \left(\int_{s}^{u} \frac{\partial B}{\partial x}\left(\theta, Z_{\theta}^{s, y}\right) d \theta\right) d u$. As the function $\frac{\partial^{2} B}{\partial x^{2}}(t, x)$ is also bounded in $[0, T] \times \mathbb{R}$, we get

$$
\left|\frac{\partial^{2} v_{t_{k}, x}}{\partial y^{2}}(s, y)\right| \leq C\left(\left(\left|g_{\varepsilon}^{\prime}\right|+g_{\varepsilon}\right) * \Gamma\left(t_{k}, \cdot ; s, y\right)\right)(x),
$$

from which we can only upper-bound quantities like $\sup _{z \in \mathbb{R}}\left\|\frac{\partial^{2} v_{t_{k}, \cdot}}{\partial z^{2}}(s, z)\right\|_{L^{1}(\mathbb{R})}$ by $C / \varepsilon^{\alpha}$ with $\alpha>0$. To prove (3.14) and (3.15), we proceed as follows: for all $(s, y) \in\left[0, t_{k}\right) \times \mathbb{R}$, we define the function $u_{t_{k}, x}(s, y)=v_{t_{k}, x}\left(t_{k}-s, y\right)$ so that $u_{t_{k}, x}(s, y)$ is the unique bounded classical solution of the Cauchy problem

$$
\left\{\begin{array}{c}
\frac{\partial u_{t_{k}, x}}{\partial s}(s, y)=\frac{1}{2} \sigma^{2} \frac{\partial^{2} u_{t_{k}, x}}{\partial y^{2}}(s, y)+B\left(t_{k}-s, y\right) \frac{\partial u_{t_{k}, x}}{\partial y}(s, y) \\
\forall(s, y) \in\left[0, t_{k}\right) \times \mathbb{R} \\
u_{t_{k}, x}(0, y)=H_{\varepsilon}(x-y), \forall y \in \mathbb{R}
\end{array}\right.
$$


We easily deduce from this equation that for all $(s, y) \in\left[0, t_{k}\right) \times \mathbb{R}$,

$$
\begin{aligned}
& u_{t_{k}, x}(s, y) \\
& \quad=S_{s} u_{t_{k}, x}(0, \cdot)(y)+\int_{0}^{s} S_{s-\theta}\left(B\left(t_{k}-\theta, \cdot\right) \frac{\partial u_{t_{k}, x}}{\partial y}(\theta, \cdot)\right)(y) d \theta \\
& \quad=\int_{y}^{+\infty} g_{\sigma^{2} s+\varepsilon}(z-x) d z+\int_{0}^{s} \int_{\mathbb{R}} g_{\sigma^{2}(s-\theta)}(y-z) B\left(t_{k}-\theta, z\right) \frac{\partial u_{t_{k}, x}}{\partial y}(\theta, z) d z d \theta .
\end{aligned}
$$

Deriving the expression above two times, we get

$$
\begin{aligned}
\frac{\partial^{2} u_{t_{k}, x}}{\partial y^{2}} & (s, y) \\
= & -g_{\sigma^{2} s+\varepsilon}^{\prime}(y-x) \\
& +\int_{0}^{s} \int_{\mathbb{R}} g_{\sigma^{2}(s-\theta)}^{\prime}(y-z) \frac{\partial}{\partial z}\left(B\left(t_{k}-\theta, z\right) \frac{\partial u_{t_{k}, x}}{\partial y}(\theta, z)\right) d z d \theta .
\end{aligned}
$$

With $|B|$ and $\left|\frac{\partial B}{\partial x}\right|$ uniformly bounded, we have

$$
\begin{aligned}
\left|\frac{\partial^{2} u_{t_{k}, x}}{\partial y^{2}}\right|(s, y) \leq & \left|g_{\sigma^{2} s+\varepsilon}^{\prime}\right|(y-x) \\
& +C \int_{0}^{s} \int_{\mathbb{R}}\left|g_{\sigma^{2}(s-\theta)}^{\prime}\right|(y-z) g_{\varepsilon+2 \sigma^{2} \theta}(x-z) d z d \theta \\
& +C \int_{0}^{s} \int_{\mathbb{R}}\left|g_{\sigma^{2}(s-\theta)}^{\prime}\right|(y-z)\left|\frac{\partial^{2} u_{t_{k}, x}}{\partial y^{2}}\right|(\theta, z) d z d \theta \\
\leq & \left|g_{\sigma^{2} s+\varepsilon}^{\prime}\right|(y-x)+C g_{2 \sigma^{2} s+\varepsilon}(y-x) \\
& +C \int_{0}^{s} \int_{\mathbb{R}}\left|g_{\sigma^{2}(s-\theta)}^{\prime}\right|(y-z)\left|\frac{\partial^{2} u_{t_{k}, x}}{\partial y^{2}}\right|(\theta, z) d z d \theta .
\end{aligned}
$$

In view of (3.16), $x \longrightarrow \frac{\partial^{2} u_{t_{k}, x}}{\partial y^{2}}(s, y)$ is in $L^{1}(\mathbb{R})$, uniformly in $y$ and $s$ and hence,

$$
\begin{aligned}
& \sup _{y \in \mathbb{R}} \int_{\mathbb{R}}\left|\frac{\partial^{2} u_{t_{k}, x}}{\partial y^{2}}\right|(s, y) d x \\
& \quad \leq \frac{C}{\sqrt{\varepsilon+\sigma^{2} s}}+\int_{0}^{s} \frac{C}{\sqrt{s-\theta}}\left(\sup _{z \in \mathbb{R}} \int_{\mathbb{R}}\left|\frac{\partial^{2} u_{t_{k}, x}}{\partial y^{2}}\right|(\theta, z) d x\right) d \theta .
\end{aligned}
$$

We apply Gronwall's lemma to get (3.14). To prove (3.15), we start from (3.17):

$$
\begin{aligned}
\left|\frac{\partial^{2} u_{t_{k}, x}}{\partial y^{2}}\right|^{5 / 4}(s, y) \leq & C\left|g_{\sigma^{2} s+\varepsilon}^{\prime}\right|^{5 / 4}(y-x)+C g_{2 \sigma^{2} s+\varepsilon}^{5 / 4}(y-x) \\
& +\left(C \int_{0}^{s} \int_{\mathbb{R}}\left|g_{\sigma^{2}(s-\theta)}^{\prime}\right|(y-z)\left|\frac{\partial^{2} u_{t_{k}, x}}{\partial y^{2}}\right|(\theta, z) d z d \theta\right)^{5 / 4} \\
\leq & \frac{C}{\left(\sigma^{2} s+\varepsilon\right)^{3 / 4}} g_{2 \sigma^{2} s+2 \varepsilon}(y-x) \\
& +\int_{0}^{s} \int_{\mathbb{R}} \frac{C}{(s-\theta)^{5 / 8}}\left|\frac{\partial^{2} u_{t_{k}, x}}{\partial y^{2}}\right|^{5 / 4}(\theta, z) g_{2 \sigma^{2}(s-\theta)}(y-z) d z d \theta .
\end{aligned}
$$


Hence,

$$
\begin{aligned}
\int_{\mathbb{R}}\left|\frac{\partial^{2} u_{t_{k}, x}}{\partial y^{2}}\right|^{5 / 4}(s, y) d y \\
\quad \leq \frac{C}{\left(\sigma^{2} s+\varepsilon\right)^{3 / 4}}+\int_{0}^{s} \frac{C}{(s-\theta)^{5 / 8}} \int_{\mathbb{R}}\left|\frac{\partial^{2} u_{t_{k}, x}}{\partial y^{2}}\right|^{5 / 4}(\theta, z) d z d \theta
\end{aligned}
$$

from which we conclude by Gronwall's lemma.

\subsection{Estimates on the smoothing error.}

Proof of Lemma 3.1. First, we observe that $\forall z \in \mathbb{R}, H_{\varepsilon}(z)=\mathbb{E} H\left(z-W_{\varepsilon}\right)$. Then, for any $x$ in $\mathbb{R}$,

$$
\begin{aligned}
\int_{\mathbb{R}}\left|H(x-z)-H_{\varepsilon}(x-z)\right| d z & \leq \mathbb{E} \int_{\mathbb{R}}\left|H(x-z)-H\left(x-z-W_{\varepsilon}\right)\right| d z \\
& =\mathbb{E}\left|W_{\varepsilon}\right|=\frac{2 \sqrt{\varepsilon}}{\sqrt{2 \pi}} .
\end{aligned}
$$

With the density $U(t, z)$ of $Z_{t}$ bounded in $z \in \mathbb{R}$, uniformly in $t$,

$\left|\mathbb{E} H\left(x-Z_{t_{k}}\right)-\mathbb{E} H_{\varepsilon}\left(x-Z_{t_{k}}\right)\right| \leq \int_{\mathbb{R}}\left|H(x-z)-H_{\varepsilon}(x-z)\right| U\left(t_{k}, z\right) d z \leq C \sqrt{\varepsilon}$,

which gives (3.8). Now, for any $x \in \mathbb{R}$ and $k \geq 1$,

$$
\begin{gathered}
\mathbb{E}\left|H\left(x-Y_{t_{k}}^{i}\right)-H_{\varepsilon}\left(x-Y_{t_{k}}^{i}\right)\right| \\
=\mathbb{E}\left(\mathbb{E}^{\mathcal{F}_{t_{k-1}} \mid H\left(x-Y_{t_{k-1}}^{i}-\Delta t A^{\prime}\left(\bar{V}_{t_{k-1}}\left(Y_{t_{k-1}}^{i}\right)\right)-\sigma W_{\Delta t}\right)}\right. \\
\left.\quad-H_{\varepsilon}\left(x-Y_{t_{k-1}}^{i}-\Delta t A^{\prime}\left(\bar{V}_{t_{k-1}}\left(Y_{t_{k-1}}^{i}\right)\right)-\sigma W_{\Delta t}\right) \mid\right) \\
=\int_{\mathbb{R}} g_{\sigma^{2} \Delta t}(z) \mathbb{E} \mid H\left(x-Y_{t_{k-1}}^{i}-\Delta t A^{\prime}\left(\bar{V}_{t_{k-1}}\left(Y_{t_{k-1}}^{i}\right)\right)-z\right) \\
\quad-H_{\varepsilon}\left(x-Y_{t_{k-1}}^{i}-\Delta t A^{\prime}\left(\bar{V}_{t_{k-1}}\left(Y_{t_{k-1}}^{i}\right)\right)-z\right) \mid d z \\
\leq C \frac{\sqrt{\varepsilon}}{\sqrt{\Delta t}} .
\end{gathered}
$$

Similarly, for $i \neq j$, with the Brownian motions $\left(W^{i}\right)$ and $\left(W^{j}\right)$ independent,

$$
\begin{gathered}
\mathbb{E}\left|H\left(Y_{t_{k}}^{j}-Y_{t_{k}}^{i}\right)-H_{\varepsilon}\left(Y_{t_{k}}^{j}-Y_{t_{k}}^{i}\right)\right| \\
=\int_{\mathbb{R}} g_{2 \sigma^{2} \Delta t}(z) \mathbb{E} \mid H\left(Y_{t_{k-1}}^{j}+\Delta t A^{\prime}\left(\bar{V}_{t_{k-1}}\left(Y_{t_{k-1}}^{j}\right)\right)\right. \\
\left.-Y_{t_{k-1}}^{i}-\Delta t A^{\prime}\left(\bar{V}_{t_{k-1}}\left(Y_{t_{k-1}}^{i}\right)\right)-z\right) \\
-H_{\varepsilon}\left(Y_{t_{k-1}}^{j}+\Delta t A^{\prime}\left(\bar{V}_{t_{k-1}}\left(Y_{t_{k-1}}^{j}\right)\right)\right. \\
\left.\quad-Y_{t_{k-1}}^{i}-\Delta t A^{\prime}\left(\bar{V}_{t_{k-1}}\left(Y_{t_{k-1}}^{i}\right)\right)-z\right) \mid d z \\
\leq C \frac{\sqrt{\varepsilon}}{\sqrt{\Delta t}}
\end{gathered}
$$

from which we deduce (3.9). 


\subsection{Estimates on the initialization error.}

Proof of Lemma 3.2. For all $t>0$, the function $y \longrightarrow \mathbb{E} H_{\varepsilon}\left(x-Z_{t}^{0, y}\right)$ is differentiable and $\frac{\partial}{\partial y} \mathbb{E} H_{\varepsilon}\left(x-Z_{t}^{0, y}\right)=-\mathbb{E}\left(g_{\varepsilon}\left(x-Z_{t}^{0, y}\right) \frac{d Z_{t}^{0, y}}{d y}\right)$, where $\frac{d Z_{t}^{0, y}}{d y}=$ $\exp \left(\int_{0}^{t} \frac{\partial B}{\partial x}\left(s, Z_{s}^{0, y}\right) d s\right) \leq C$. By integration by parts,

$$
\begin{aligned}
\int_{\mathbb{R}} \mathbb{E} H_{\varepsilon}\left(x-Z_{t}^{0, y}\right) m_{0}(d y)= & \mathbb{E} H_{\varepsilon}\left(x-Z_{t}^{0,0}\right)-\int_{-\infty}^{0} \frac{\partial}{\partial y} \mathbb{E} H_{\varepsilon}\left(x-Z_{t}^{0, y}\right) V_{0}(y) d y \\
& +\int_{0}^{+\infty} \frac{\partial}{\partial y} \mathbb{E} H_{\varepsilon}\left(x-Z_{t}^{0, y}\right)\left(1-V_{0}(y)\right) d y .
\end{aligned}
$$

Similarly,

$$
\begin{aligned}
& \int_{\mathbb{R}} \mathbb{E} H_{\varepsilon}\left(x-Z_{t}^{0, y}\right) \bar{m}_{0}(d y) \\
& \quad=\int_{-\infty}^{0} \mathbb{E} H_{\varepsilon}\left(x-Z_{t}^{0, y}\right) d \bar{V}_{0}(y)-\int_{0}^{+\infty} \mathbb{E} H_{\varepsilon}\left(x-Z_{t}^{0, y}\right) d\left(1-\bar{V}_{0}(y)\right)
\end{aligned}
$$

and the integration by parts formula for a Stieltjes integral gives

$$
\begin{aligned}
\int_{\mathbb{R}} \mathbb{E} H_{\varepsilon}\left(x-Z_{t}^{0, y}\right) \bar{m}_{0}(d y)= & \mathbb{E} H_{\varepsilon}\left(x-Z_{t}^{0,0}\right)-\int_{-\infty}^{0} \frac{\partial}{\partial y} \mathbb{E} H_{\varepsilon}\left(x-Z_{t}^{0, y}\right) \bar{V}_{0}(y) d y \\
& +\int_{0}^{+\infty} \frac{\partial}{\partial y} \mathbb{E} H_{\varepsilon}\left(x-Z_{t}^{0, y}\right)\left(1-\bar{V}_{0}(y)\right) d y .
\end{aligned}
$$

Thus, we obtain the following expression for the initialization error

$$
\begin{gathered}
\int_{\mathbb{R}} \mathbb{E} H_{\varepsilon}\left(x-Z_{t}^{0, y}\right) m_{0}(d y)-\int_{\mathbb{R}} \mathbb{E} H_{\varepsilon}\left(x-Z_{t}^{0, y}\right) \bar{m}_{0}(d y) \\
=\int_{\mathbb{R}} \frac{\partial}{\partial y} \mathbb{E} H_{\varepsilon}\left(x-Z_{t}^{0, y}\right)\left(\bar{V}_{0}(y)-V_{0}(y)\right) d y,
\end{gathered}
$$

from which we deduce that

$$
\begin{aligned}
\sup _{x \in \mathbb{R}}\left|\int_{\mathbb{R}} \mathbb{E} H_{\varepsilon}\left(x-Z_{t_{k}}^{0, y}\right) m_{0}(d y)-\int_{\mathbb{R}} \mathbb{E} H_{\varepsilon}\left(x-Z_{t_{k}}^{0, y}\right) \bar{m}_{0}(d y)\right| \\
\leq C\left\|V_{0}-\bar{V}_{0}\right\|_{L^{\infty}(\mathbb{R})} \sup _{x \in \mathbb{R}} \int_{\mathbb{R}} \mathbb{E} g_{\varepsilon}\left(x-Z_{t_{k}}^{0, y}\right) d y
\end{aligned}
$$

and

$$
\begin{aligned}
& \left\|\int_{\mathbb{R}} \mathbb{E} H_{\varepsilon}\left(x-Z_{t_{k}}^{0, y}\right) m_{0}(d y)-\int_{\mathbb{R}} \mathbb{E} H_{\varepsilon}\left(x-Z_{t_{k}}^{0, y}\right) \bar{m}_{0}(d y)\right\|_{L^{1}(\mathbb{R})} \\
& \leq C\left\|V_{0}-\bar{V}_{0}\right\|_{L^{1}(\mathbb{R})} \sup _{y \in \mathbb{R}} \int_{\mathbb{R}} \mathbb{E} g_{\varepsilon}\left(x-Z_{t_{k}}^{0, y}\right) d x .
\end{aligned}
$$

In view of Lemma 3.8 the exponential bound for the density of $Z_{t}^{0, y}$ gives

$$
\mathbb{E} g_{\varepsilon}\left(x-Z_{t_{k}}^{0, y}\right) \leq C g_{\varepsilon+2 \sigma^{2} t_{k}}(x-y),
$$

from which we easily conclude. 


\subsection{Estimates on the statistical error.}

Proof of Lemma 3.3 . We consider the statistical error

$$
\mathbb{E}\left|\frac{1}{N} \sum_{i=1}^{N} \int_{0}^{t_{k}} \sigma \frac{\partial v_{t_{k}, x}}{\partial y}\left(s, Y_{s}^{i}\right) d W_{s}^{i}\right|
$$

From (3.13),$\frac{\partial v_{t_{k}, x}}{\partial y}(s, y)$ is uniformly bounded on $\left[0, t_{k}\right] \times \mathbb{R}$ by $C / \sqrt{\varepsilon}$. Then, by the Cauchy-Schwarz inequality, for all $x \in \mathbb{R}$,

$$
\begin{aligned}
\mathbb{E}\left|\frac{1}{N} \sum_{i=1}^{N} \int_{0}^{t_{k}} \sigma \frac{\partial v_{t_{k}, x}}{\partial y}\left(s, Y_{s}^{i}\right) d W_{s}^{i}\right| & \leq \sqrt{\mathbb{E}\left(\frac{1}{N} \sum_{i=1}^{N} \int_{0}^{t_{k}} \sigma \frac{\partial v_{t_{k}, x}}{\partial y}\left(s, Y_{s}^{i}\right) d W_{s}^{i}\right)^{2}} \\
& \leq \sqrt{\frac{1}{N^{2}} \sum_{i=1}^{N} \int_{0}^{t_{k}} \sigma^{2} \mathbb{E}\left(\frac{\partial v_{t_{k}, x}}{\partial y}\left(s, Y_{s}^{i}\right)\right)^{2} d s}
\end{aligned}
$$

For each $i$ in $\{1, \ldots, N\}$, let $\left(\mathcal{Z}_{t}^{i}\right)_{0 \leq t \leq T}$ be defined by

$$
\mathcal{Z}_{t}^{i}=\exp \left(\int_{0}^{t} \frac{A^{\prime}\left(\bar{V}_{\eta(s)}\left(Y_{\eta(s)}^{i}\right)\right)}{\sigma^{2}} d Y_{s}^{i}-\frac{1}{2} \int_{0}^{t} \frac{\left(A^{\prime}\left(\bar{V}_{\eta(s)}\left(Y_{\eta(s)}^{i}\right)\right)\right)^{2}}{\sigma^{2}} d s\right) .
$$

By the Girsanov theorem, under the probability $\mathbb{Q}^{i}$ such that $\left.\left(d \mathbb{Q}^{i} / d \mathbb{P}\right)\right|_{\mathcal{F}_{t}}=1 / \mathcal{Z}_{t}^{i}$, $\left(Y_{t}^{i} / \sigma\right)_{0 \leq t \leq T}$ is a one-dimensional Brownian motion on $\left(\Omega, \mathcal{F}_{T}, \mathbb{Q}^{i}\right)$, starting at $y_{0}^{i} / \sigma$ and

$$
\mathbb{E}\left(\frac{\partial v_{t_{k}, x}}{\partial y}\left(s, Y_{s}^{i}\right)\right)^{2}=\mathbb{E}^{\mathbb{Q}^{i}}\left[\left(\frac{\partial v_{t_{k}, x}}{\partial y}\left(s, Y_{s}^{i}\right)\right)^{2} \mathcal{Z}_{s}^{i}\right]
$$

where $\mathbb{E}^{\mathbb{Q}^{i}}$ denotes the expectation under $\mathbb{Q}^{i}$. Moreover,

$$
\mathbb{E}^{\mathbb{Q}^{i}}\left(\mathcal{Z}_{s}^{i 2}\right) \leq \exp \left(\frac{s}{\sigma^{2}} \sup _{v \in[0,1]}\left|A^{\prime}(v)\right|^{2}\right) \leq C .
$$

Using the Cauchy-Schwarz inequality and Lemma 3.9,

$$
\begin{aligned}
\mathbb{E}\left(\frac{\partial v_{t_{k}, x}}{\partial y}\left(s, Y_{s}^{i}\right)\right)^{2} & \leq C \sqrt{\mathbb{E}\left(\frac{\partial v_{t_{k}, x}}{\partial y}\left(s, y_{0}^{i}+\sigma W_{s}\right)\right)^{4}} \\
& \leq C \sqrt{\left(g_{\varepsilon+2 \sigma^{2}\left(t_{k}-s\right)}^{4} * g_{\sigma^{2} s}\right)\left(x-y_{0}^{i}\right)}
\end{aligned}
$$

An easy computation shows that for any $z \in \mathbb{R}$,

$$
\sqrt{g_{\varepsilon+2 \sigma^{2}\left(t_{k}-s\right)}^{4} * g_{\sigma^{2} s}(z)} \leq \frac{C}{t_{k}^{1 / 4}\left(t_{k}-s\right)^{3 / 4}} \phi(z)
$$


where the function $\phi$ is defined on $\mathbb{R}$ by $\phi(z)=\exp \left(-z^{2} /\left(\varepsilon+4 \sigma^{2} t_{k}\right)\right)$. Finally, for all $x \in \mathbb{R}$,

$$
\begin{aligned}
\mathbb{E}\left|\frac{1}{N} \sum_{i=1}^{N} \int_{0}^{t_{k}} \sigma \frac{\partial v_{t_{k}, x}}{\partial y}\left(s, Y_{s}^{i}\right) d W_{s}^{i}\right| & \leq \frac{C}{\sqrt{N}} \sqrt{\frac{1}{N} \sum_{i=1}^{N} \phi\left(x-y_{0}^{i}\right) \frac{1}{t_{k}^{1 / 4}} \int_{0}^{t_{k}} \frac{1}{\left(t_{k}-s\right)^{3 / 4}}} \\
& \leq \frac{C}{\sqrt{N}} \sqrt{\frac{1}{N} \sum_{i=1}^{N} \phi\left(x-y_{0}^{i}\right)}=\frac{C}{\sqrt{N}} \sqrt{\phi * \overline{m_{0}}(x)} .
\end{aligned}
$$

Thus,

$$
\sup _{x \in \mathbb{R}} \mathbb{E}\left|\frac{1}{N} \sum_{i=1}^{N} \int_{0}^{t_{k}} \sigma \frac{\partial v_{t_{k}, x}}{\partial y}\left(s, Y_{s}^{i}\right) d W_{s}^{i}\right| \leq C / \sqrt{N}
$$

and

$$
\mathbb{E}\left\|\frac{1}{N} \sum_{i=1}^{N} \int_{0}^{t_{k}} \sigma \frac{\partial v_{t_{k}, \cdot}}{\partial y}\left(s, Y_{s}^{i}\right) d W_{s}^{i}\right\|_{L^{1}(\mathbb{R})} \leq \frac{C}{\sqrt{N}} \int_{\mathbb{R}} \sqrt{\phi * \bar{m}_{0}(x)} d x .
$$

To end the proof, we decompose the integral above into three parts:

$$
\begin{aligned}
\int_{\mathbb{R}} & \sqrt{\phi * \bar{m}_{0}(x)} d x \\
& =\int_{-\infty}^{\underline{y}_{0}} \sqrt{\phi * \bar{m}_{0}(x)} d x+\int_{\underline{y}_{0}}^{\bar{y}_{0}} \sqrt{\phi * \bar{m}_{0}(x)} d x+\int_{\bar{y}_{0}}^{+\infty} \sqrt{\phi * \bar{m}_{0}(x)} d x,
\end{aligned}
$$

where $\underline{y}_{0}=\min _{\{1 \leq i \leq N\}} y_{0}^{i}$ and $\bar{y}_{0}=\max _{\{1 \leq i \leq N\}} y_{0}^{i}$, so that

$$
\int_{-\infty}^{\underline{y}_{0}} \sqrt{\phi * \bar{m}_{0}(x)} d x+\int_{\bar{y}_{0}}^{+\infty} \sqrt{\phi * \bar{m}_{0}(x)} \leq \int_{\mathbb{R}} \sqrt{\phi(x)} d x \leq C .
$$

Now, we note that $\phi * \bar{m}_{0}(x)=\phi * m_{0}(x)+\phi^{\prime} *\left(V_{0}-\bar{V}_{0}\right)(x)$ and

$$
\int_{\underline{y}_{0}}^{\bar{y}_{0}} \sqrt{\phi * \bar{m}_{0}(x)} d x \leq \int_{\mathbb{R}} \sqrt{\phi * m_{0}(x)} d x+\int_{\underline{y}_{0}}^{\bar{y}_{0}} \sqrt{\left|\phi^{\prime} *\left(V_{0}-\bar{V}_{0}\right)(x)\right|} d x .
$$

We upper-bound $\int_{\mathbb{R}} \sqrt{\phi * m_{0}(x)} d x$ by using Hypothesis (H3)(ii): there exist constants $M>0, \eta \geq 0$ and $\alpha>0$ such that

$$
\mathbb{1}_{[-M, M]^{c}} m_{0}(d x) \leq \mathbb{1}_{[-M, M]^{c}} \eta \exp \left(-\alpha x^{2} / 2\right) d x .
$$

Then,

$$
\begin{aligned}
\int_{\mathbb{R}} \sqrt{\phi} & * m_{0}(x) \\
\leq & \int_{-\infty}^{-M} \sqrt{\eta\left(\phi * e^{-\alpha \frac{(\cdot)^{2}}{2}}\right)(x)} d x+2 M \sqrt{\|\phi\|_{L^{\infty}(\mathbb{R})}} \\
& +\int_{M}^{+\infty} \sqrt{\eta\left(\phi * e^{-\alpha \frac{(\cdot)^{2}}{2}}\right)(x)} d x .
\end{aligned}
$$

As $\left(\phi * \exp \left(-\alpha(\cdot)^{2} / 2\right)\right)(x) \leq \sqrt{\pi \alpha} \exp \left(-\alpha x^{2} /\left(2+2 \alpha\left(\varepsilon+4 \sigma^{2} t_{k}\right)\right)\right)$, we have that

$$
\int_{-\infty}^{-M} \sqrt{\eta \phi * e^{-\alpha \cdot \frac{(\cdot)^{2}}{2}}(x)} d x+\int_{M}^{+\infty} \sqrt{\eta \phi * e^{-\alpha \frac{(\cdot)^{2}}{2}}(x)} d x \leq C
$$


and thus $\int_{\underline{y}_{0}}^{\bar{y}_{0}} \sqrt{\phi * m_{0}(x)} d x \leq C$ (the constant $C$ depends on $T$ but does not depend on $t_{k}$ ). Moreover, $\left\|\phi^{\prime}\right\|_{L^{1}(\mathbb{R})} \leq C$ (independent of $t_{k}$ ) and $\left\|V_{0}-\bar{V}_{0}\right\|_{L^{\infty}(\mathbb{R})} \leq 1 / N$. Then

$$
\int_{\underline{y}_{0}}^{\bar{y}_{0}} \sqrt{\left|\phi^{\prime} *\left(V_{0}-\bar{V}_{0}\right)(x)\right|} d x \leq C\left(\left|\underline{y}_{0}\right|+\left|\bar{y}_{0}\right|\right) \sqrt{1 / N}
$$

By construction of the $\left(y_{0}^{i}\right)$ and thanks to Hypothesis (H3)(ii), one can see easily that $\left(\left|\underline{y}_{0}\right|+\left|\bar{y}_{0}\right|\right) \leq C \sqrt{\ln (N)}$, which concludes the proof.

3.5. Proof of Lemma 3.4: Estimates for the time discretization error. We consider now the main part of the error in the decomposition (3.7). We split it into two parts, making apparent the difference between the drift functions $B$ at the discrete times $t_{l}$ and its approximation $A^{\prime}\left(\bar{V}_{t_{l}}(x)\right)$ :

$$
\begin{aligned}
\mathbb{E}\left|\frac{1}{N} \sum_{i=1}^{N} \sum_{l=0}^{k-1} \int_{t_{l}}^{t_{l+1}} \frac{\partial v_{t_{k}, x}}{\partial y}\left(s, Y_{s}^{i}\right)\left(B\left(s, Y_{s}^{i}\right)-A^{\prime}\left(\bar{V}_{t_{l}}\left(Y_{t_{l}}^{i}\right)\right)\right) d s\right| \\
\leq \mathbb{E}\left|\frac{1}{N} \sum_{i=1}^{N} \sum_{l=0}^{k-1} \int_{t_{l}}^{t_{l+1}} \frac{\partial v_{t_{k}, x}}{\partial y}\left(s, Y_{s}^{i}\right)\left(B\left(s, Y_{s}^{i}\right)-B\left(t_{l}, Y_{t_{l}}^{i}\right)\right) d s\right| \\
\quad+\mathbb{E}\left|\frac{1}{N} \sum_{i=1}^{N} \sum_{l=0}^{k-1} \int_{t_{l}}^{t_{l+1}} \frac{\partial v_{t_{k}, x}}{\partial y}\left(s, Y_{s}^{i}\right)\left(B\left(t_{l}, Y_{t_{l}}^{i}\right)-A^{\prime}\left(\bar{V}_{t_{l}}\left(Y_{t_{l}}^{i}\right)\right)\right) d s\right| \\
:=T_{1}(x)+T_{2}(x) .
\end{aligned}
$$

We treat $T_{1}(x)$ and $T_{2}(x)$ separately.

Upper bound for $T_{1}(x)$ : this first term is a time discretization error. To obtain an error bound of order $\mathcal{O}(\Delta t)$, we need to introduce an expectation inside the absolute value in the expression of $T_{1}(x)$. For all $l$ in $\{0, \ldots, K\}$, we set $\mathcal{F}_{t_{l}}=$ $\sigma\left(W_{s}^{i} ; 0 \leq s \leq t_{l}, i=1, \ldots, N\right)$. For all $s \in\left[t_{l}, t_{l+1}\right)$, the variables $\left(R_{t_{l}, s}^{i}:=\right.$ $\left.\frac{\partial v_{t_{k}, x}}{\partial y}\left(s, Y_{s}^{i}\right)\left(B\left(s, Y_{s}^{i}\right)-B\left(t_{l}, Y_{t_{l}}^{i}\right)\right), i=1, \ldots, N\right)$ are $\mathcal{F}_{t_{l}}$-conditionally independent. Hence,

$$
\begin{aligned}
\mathbb{E}\left|\frac{1}{N} \sum_{i=1}^{N} R_{t_{l}, s}^{i}-\mathbb{E}^{\mathcal{F}_{t_{l}}}\left(R_{t_{l}, s}^{i}\right)\right| & \leq \frac{1}{\sqrt{N}} \sqrt{\frac{1}{N} \sum_{i=1}^{N} \mathbb{E}\left(R_{t_{l}, s}^{i}\right)^{2}} \\
& \leq \frac{C}{\sqrt{N}} \sqrt{\frac{1}{N} \sum_{i=1}^{N} \mathbb{E}\left(\frac{\partial v_{t_{k}, x}}{\partial y}\left(s, Y_{s}^{i}\right)\right)^{2}} .
\end{aligned}
$$

Thus, we isolate a statistical error in $T_{1}(x)$ :

$$
\begin{aligned}
T_{1}(x) \leq \mathbb{E} \mid \frac{1}{N} \sum_{i=1}^{N} \sum_{l=0}^{k-1} \int_{t_{l}}^{t_{l+1}} \mathbb{E}^{\mathcal{F}_{t_{l}}\left\{\frac{\partial v_{t_{k}, x}}{\partial y}\left(s, Y_{s}^{i}\right)\left(B\left(s, Y_{s}^{i}\right)-B\left(t_{l}, Y_{t_{l}}^{i}\right)\right)\right\} d s \mid} \\
+\frac{C}{\sqrt{N}} \int_{0}^{t_{k}} \sqrt{\frac{1}{N} \sum_{i=1}^{N} \mathbb{E}\left(\frac{\partial v_{t_{k}, x}}{\partial y}\left(s, Y_{s}^{i}\right)\right)^{2}} d s .
\end{aligned}
$$


By Itô's formula,

$$
\begin{aligned}
\mathbb{E}^{\mathcal{F}_{t_{l}}}\left\{\frac{\partial v_{t_{k}, x}}{\partial y}\left(s, Y_{s}^{i}\right)\left(B\left(s, Y_{s}^{i}\right)-B\left(t_{l}, Y_{t_{l}}^{i}\right)\right)\right\} \\
=\mathbb{E}^{\mathcal{F}_{t_{l}}} \int_{t_{l}}^{s}\left[\frac{\partial}{\partial \theta}+A^{\prime}\left(\bar{V}_{t_{l}}\left(Y_{t_{l}}^{i}\right)\right) \frac{\partial}{\partial y}+\frac{\sigma^{2}}{2} \frac{\partial^{2}}{\partial y^{2}}\right]\left(\frac{\partial v_{t_{k}, x}}{\partial y}\left(B-B\left(t_{l}, Y_{t_{l}}^{i}\right)\right)\right)\left(\theta, Y_{\theta}^{i}\right) d \theta \\
=\mathbb{E}^{\mathcal{F}_{t_{l}}} \int_{t_{l}}^{s}\left[\frac{\partial v_{t_{k}, x}}{\partial y}\left(\frac{\partial B}{\partial \theta}+\frac{\sigma^{2}}{2} \frac{\partial^{2} B}{\partial y^{2}}+\left(A^{\prime}\left(\bar{V}_{t_{l}}\left(Y_{t_{l}}^{i}\right)\right)+B\left(t_{l}, Y_{t_{l}}^{i}\right)-B\right) \frac{\partial B}{\partial y}\right)\right. \\
\left.+\frac{\partial^{2} v_{t_{k}, x}}{\partial y^{2}}\left(\sigma^{2} \frac{\partial B}{\partial y}-\left(B-B\left(t_{l}, Y_{t_{l}}^{i}\right)\right)\left(B-A^{\prime}\left(\bar{V}_{t_{l}}\left(Y_{t_{l}}^{i}\right)\right)\right)\right)\right]\left(\theta, Y_{\theta}^{i}\right) d \theta .
\end{aligned}
$$

The last identity is obtained by using (3.6). As $B(s, y)$ has uniformly bounded derivatives, we obtain that

$$
\begin{aligned}
\mathbb{E} \int_{t_{l}}^{t_{l+1}} & \left|\mathbb{E}^{\mathcal{F}_{t_{l}}}\left\{\frac{\partial v_{t_{k}, x}}{\partial y}\left(s, Y_{s}^{i}\right)\left(B\left(s, Y_{s}^{i}\right)-B\left(t_{l}, Y_{t_{l}}^{i}\right)\right)\right\}\right| d s \\
\leq & C \int_{t_{l}}^{t_{l+1}} \int_{t_{l}}^{s} \mathbb{E}\left[\left|\frac{\partial v_{t_{k}, x}}{\partial y}\right|\left(\theta, Y_{\theta}^{i}\right)+\left|\frac{\partial^{2} v_{t_{k}, x}}{\partial y^{2}}\right|\left(\theta, Y_{\theta}^{i}\right)\right] d \theta d s \\
\leq & C \Delta t \int_{t_{l}}^{t_{l+1}} \mathbb{E}\left[\left|\frac{\partial v_{t_{k}, x}}{\partial y}\right|\left(s, Y_{s}^{i}\right)+\left|\frac{\partial^{2} v_{t_{k}, x}}{\partial y^{2}}\right|\left(s, Y_{s}^{i}\right)\right] d s,
\end{aligned}
$$

and

$$
\begin{aligned}
T_{1}(x) \leq & C \Delta t \int_{0}^{t_{k}} \frac{1}{N} \sum_{i=1}^{N} \mathbb{E}\left[\left(\left|\frac{\partial v_{t_{k}, x}}{\partial y}\right|+\left|\frac{\partial^{2} v_{t_{k}, x}}{\partial y^{2}}\right|\right)\left(s, Y_{s}^{i}\right)\right] d s \\
& +\frac{C}{\sqrt{N}} \int_{0}^{t_{k}} \sqrt{\frac{1}{N} \sum_{i=1}^{N} \mathbb{E}\left(\frac{\partial v_{t_{k}, x}}{\partial y}\left(s, Y_{s}^{i}\right)\right)^{2}} d s .
\end{aligned}
$$

We want to upper-bound $\left\|T_{1}(\cdot)\right\|_{L^{1}(\mathbb{R})}$ and $\sup _{x \in \mathbb{R}} T_{1}(x)$. From the proof of Lemma 3.3. we easily deduce that

$$
\begin{aligned}
\sup _{x \in \mathbb{R}} & {\left[\int_{0}^{t_{k}} \sqrt{\frac{1}{N} \sum_{i=1}^{N} \mathbb{E}\left(\frac{\partial v_{t_{k}, x}}{\partial y}\left(s, Y_{s}^{i}\right)\right)^{2}} d s\right] } \\
& +\int_{\mathbb{R}} \int_{0}^{t_{k}} \sqrt{\frac{1}{N} \sum_{i=1}^{N} \mathbb{E}\left(\frac{\partial v_{t_{k}, x}}{\partial y}\left(s, Y_{s}^{i}\right)\right)^{2}} d s d x
\end{aligned}
$$

is bounded by a positive constant $C$ depending only in $\sigma, T, A$ and $V_{0}$. Moreover, by Lemma 3.9

$$
\int_{\mathbb{R}}\left[\left|\frac{\partial v_{t_{k}, x}}{\partial y}\right|\left(s, Y_{s}^{i}\right)+\left|\frac{\partial^{2} v_{t_{k}, x}}{\partial y^{2}}\right|\left(s, Y_{s}^{i}\right)\right] d x \leq \frac{C}{\sqrt{t_{k}-s}} .
$$

Hence, we obtain that

$$
\left\|T_{1}(\cdot)\right\|_{L^{1}(\mathbb{R})} \leq C\left(\Delta t+\frac{1}{\sqrt{N}}\right) .
$$


Still by Lemma 3.9, we observe that $\sup _{x \in \mathbb{R}}\left|\frac{\partial v_{t_{k}, x}}{\partial y}\right|\left(s, Y_{s}^{i}\right) \leq C / \sqrt{t_{k}-s}$. It remains to bound $\sup _{x \in \mathbb{R}} \mathbb{E}\left(\left|\frac{\partial^{2} v_{t_{h}, x}}{\partial y^{2}}\right|\left(s, Y_{s}^{i}\right)\right)$ : let $\left(\mathcal{Z}^{i}\right)$ be the exponential martingale defined in (3.18), under the probability $\mathbb{Q}^{i}$ such that $\left.\frac{d \mathbb{Q}^{i}}{d \mathbb{P}}\right|_{\mathcal{F}_{t}}=\frac{1}{\mathcal{Z}_{t}^{i}}$. By the Girsanov theorem and the Cauchy-Schwarz inequality,

$$
\begin{aligned}
\mathbb{E}\left(\left|\frac{\partial^{2} v_{t_{k}, x}}{\partial y^{2}}\right|\left(s, Y_{s}^{i}\right)\right) & =\mathbb{E}^{\mathbb{Q}_{i}}\left(\mathcal{Z}_{s}^{i}\left|\frac{\partial^{2} v_{t_{k}, x}}{\partial y^{2}}\right|\left(s, Y_{s}^{i}\right)\right) \\
& \leq C\left(\mathbb{E}\left|\frac{\partial^{2} v_{t_{k}, x}}{\partial y^{2}}\right|^{5 / 4}\left(s, y_{0}^{i}+\sigma W_{s}\right)\right)^{4 / 5} \\
& \leq \frac{C}{s^{4 / 10}}\left\|\left|\frac{\partial^{2} v_{t_{k}, x}}{\partial y^{2}}\right|^{5 / 4}(s, \cdot)\right\|_{L^{1}(\mathbb{R})}^{4 / 5}
\end{aligned}
$$

Using (3.15), we obtain that $\sup _{x \in \mathbb{R}} \mathbb{E}\left(\left|\frac{\partial^{2} v_{t_{k}, x}}{\partial y^{2}}\right|\left(s, Y_{s}^{i}\right)\right) \leq C /\left(s^{4 / 10}\left(t_{k}-s\right)^{3 / 5}\right)$ and hence,

$$
\sup _{x \in \mathbb{R}} T_{1}(x) \leq C\left(\Delta t+\frac{1}{\sqrt{N}}\right)
$$

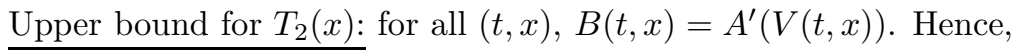

$T_{2}(x) \leq \sup _{v \in[0,1]}\left|A^{\prime \prime}(v)\right| \frac{1}{N} \sum_{i=1}^{N} \sum_{l=0}^{k-1} \mathbb{E} \int_{t_{l}}^{t_{l+1}}\left|\frac{\partial v_{t_{k}, x}}{\partial y}\left(s, Y_{s}^{i}\right)\right|\left|V\left(t_{l}, Y_{t_{l}}^{i}\right)-\bar{V}_{t_{l}}\left(Y_{t_{l}}^{i}\right)\right| d s$.

By Lemma 3.9. $\sup _{x \in \mathbb{R}}\left\|\frac{\partial v_{t_{k}, x}}{\partial z}(s, \cdot)\right\|_{L^{\infty}(\mathbb{R})}+\sup _{z \in \mathbb{R}}\left\|\frac{\partial v_{t_{k}, \cdot}}{\partial z}(s, z)\right\|_{L^{1}(\mathbb{R})}$ is bounded by $C / \sqrt{t_{k}-s}$. Then,

$$
\begin{array}{rl}
\sup _{x \in \mathbb{R}} & \mathbb{E} T_{2}(x)+\mathbb{E}\left\|T_{2}(\cdot)\right\|_{L^{1}(\mathbb{R})} \\
& \leq \sum_{l=0}^{k-1} \frac{C \Delta t}{\sqrt{t_{k}-t_{l}}} \frac{1}{N} \sum_{i=1}^{N} \mathbb{E}\left|V\left(t_{l}, Y_{t_{l}}^{i}\right)-\bar{V}_{t_{l}}\left(Y_{t_{l}}^{i}\right)\right| .
\end{array}
$$

Now, the estimation of $T_{2}$ is based on the upper bound of terms of the sequence

$$
\left(\frac{1}{N} \sum_{i=1}^{N} \mathbb{E}\left|V\left(t_{l}, Y_{t_{l}}^{i}\right)-\bar{V}_{t_{l}}\left(Y_{t_{l}}^{i}\right)\right|\right)_{l=1, \ldots, k-1} .
$$

To obtain an induction formula on this sequence we introduce a new family of discrete time processes. For each $i$ in $\{1, \ldots, N\}$, we denote by $\left(\bar{Z}_{t_{k}}^{i}, k=0, \ldots, K\right)$ the discrete-time process solution of

$$
\left\{\begin{array}{l}
\bar{Z}_{t_{k+1}}^{i}=\bar{Z}_{t_{k}}^{i}+\Delta t B\left(t_{k}, \bar{Z}_{t_{k}}^{i}\right)+\sigma\left(W_{t_{k+1}}^{i}-W_{t_{k}}^{i}\right), \\
\bar{Z}_{0}^{i}=y_{0}^{i} .
\end{array}\right.
$$


With the function $V$ uniformly Lipschitz, we remark that

$$
\begin{aligned}
\left|V\left(t_{l}, Y_{t_{l}}^{i}\right)-V\left(t_{l}, \bar{Z}_{t_{l}}^{i}\right)\right| & \leq C\left|Y_{t_{l}}^{i}-\bar{Z}_{t_{l}}^{i}\right| \\
& \leq C\left|\Delta t \sum_{m=0}^{l-1} A^{\prime}\left(\bar{V}_{t_{m}}\left(Y_{t_{m}}^{i}\right)\right)-A^{\prime}\left(V\left(t_{m}, \bar{Z}_{t_{m}}^{i}\right)\right)\right| \\
& \leq C \Delta t \sum_{m=0}^{l-1}\left|V\left(t_{m}, \bar{Z}_{t_{m}}^{i}\right)-\bar{V}_{t_{m}}\left(Y_{t_{m}}^{i}\right)\right| .
\end{aligned}
$$

Then,

$$
\begin{aligned}
\frac{1}{N} \sum_{i=1}^{N} \mathbb{E}\left|V\left(t_{l}, Y_{t_{l}}^{i}\right)-\bar{V}_{t_{l}}\left(Y_{t_{l}}^{i}\right)\right| \leq & \frac{1}{N} \sum_{i=1}^{N} \mathbb{E}\left|V\left(t_{l}, \bar{Z}_{t_{l}}^{i}\right)-\bar{V}_{t_{l}}\left(Y_{t_{l}}^{i}\right)\right| \\
& +C \Delta t \sum_{m=0}^{l-1} \frac{1}{N} \sum_{i=1}^{N} \mathbb{E}\left|V\left(t_{m}, \bar{Z}_{t_{m}}^{i}\right)-\bar{V}_{t_{m}}\left(Y_{t_{m}}^{i}\right)\right|
\end{aligned}
$$

For all $l$ in $\{1, \ldots, K\}$, we define

$$
\bar{E}_{t_{l}}:=\frac{1}{N} \sum_{i=1}^{N} \mathbb{E}\left|V\left(t_{l}, \bar{Z}_{t_{l}}^{i}\right)-\bar{V}_{t_{l}}\left(Y_{t_{l}}^{i}\right)\right|
$$

Thus, we have

$$
\frac{1}{N} \sum_{i=1}^{N} \mathbb{E}\left|V\left(t_{l}, Y_{t_{l}}^{i}\right)-\bar{V}_{t_{l}}\left(Y_{t_{l}}^{i}\right)\right| \leq \bar{E}_{t_{l}}+C \Delta t \sum_{m=0}^{l-1} \bar{E}_{t_{m}} .
$$

An induction relation for $\left(\bar{E}_{t_{l}}, l=0, \ldots, K\right)$ is given in the following

Lemma 3.10. Assume (H1), (H2) and (H3). For $l=0, \ldots, K$, one has

$$
\bar{E}_{t_{l}} \leq \sum_{n=0}^{l-1} \frac{C \Delta t}{\sqrt{t_{l}-t_{n}}} \bar{E}_{t_{n}}+C\left(\Delta t+\left\|V_{0}-\bar{V}_{0}\right\|_{L^{\infty}(\mathbb{R})}+\frac{1}{\sqrt{N}}\right)
$$

and by Gronwall's lemma,

$$
\bar{E}_{t_{l}} \leq C\left(\Delta t+\left\|V_{0}-\bar{V}_{0}\right\|_{L^{\infty}(\mathbb{R})}+\frac{1}{\sqrt{N}}\right)
$$


In view of (3.22), (3.23) and this previous estimate, we obtain that

$$
\begin{aligned}
\sup _{x \in \mathbb{R}} \mathbb{E} T_{2}(x)+\mathbb{E}\left\|T_{2}(\cdot)\right\|_{L^{1}(\mathbb{R})} & \leq \sum_{l=0}^{k-1} \frac{C \Delta t}{\sqrt{t_{k}-t_{l}}}\left(\Delta t+\left\|V_{0}-\bar{V}_{0}\right\|_{L^{\infty}(\mathbb{R})}+\frac{1}{\sqrt{N}}\right) \\
& \leq C\left(\left\|V_{0}-\bar{V}_{0}\right\|_{L^{\infty}(\mathbb{R})}+\Delta t+\frac{1}{\sqrt{N}}\right) .
\end{aligned}
$$

With the estimates (3.19) and 3.21) on $T_{1}$, this ends the proof of Lemma 3.4

Proof of Lemma 3.10, First, we note that $\bar{E}_{0} \leq\left\|V_{0}-\bar{V}_{0}\right\|_{L^{\infty}(\mathbb{R})}$ and for $l=$ $1, \ldots, K$

$$
\bar{E}_{t_{l}}=\frac{1}{N} \sum_{i=1}^{N} \mathbb{E}\left|\mathbb{E} H\left(x-Z_{t_{l}}\right)\right|_{x=\bar{Z}_{t_{l}}^{i}}-\frac{1}{N} \sum_{j=1}^{N} H\left(Y_{t_{l}}^{i}-Y_{t_{l}}^{j}\right) \mid
$$

To prove the induction formula, we decompose each term $\bar{E}_{t_{l}}$ into five parts. As in the beginning of the proof of Theorem 2.2, we make apparent a smoothing error, an initialization error, a discretization error and a statistical error. First, we introduce the artificial smoothing of the Heaviside function:

$$
\begin{aligned}
\bar{E}_{t_{l}} \leq & \frac{1}{N} \sum_{i=1}^{N} \mathbb{E}\left|\mathbb{E} H\left(x-Z_{t_{l}}\right)\right|_{x=\bar{Z}_{t_{l}}^{i}}-\left.\mathbb{E} H_{\varepsilon}\left(x-Z_{t_{l}}\right)\right|_{x=\bar{Z}_{t_{l}}^{i}} \mid \\
& +\frac{1}{N} \sum_{i=1}^{N} \mathbb{E}\left|\mathbb{E} H_{\varepsilon}\left(x-Z_{t_{l}}\right)\right|_{x=\bar{Z}_{t_{l}}^{i}}-\frac{1}{N} \sum_{j=1}^{N} H_{\varepsilon}\left(Y_{t_{l}}^{i}-Y_{t_{l}}^{j}\right) \mid \\
& +\frac{1}{N} \sum_{i=1}^{N} \mathbb{E}\left|\frac{1}{N} \sum_{j=1}^{N} H_{\varepsilon}\left(Y_{t_{l}}^{i}-Y_{t_{l}}^{j}\right)-\frac{1}{N} \sum_{j=1}^{N} H\left(Y_{t_{l}}^{i}-Y_{t_{l}}^{j}\right)\right|
\end{aligned}
$$

and by Lemma 3.1 .

$\bar{E}_{t_{l}} \leq \frac{1}{N} \sum_{i=1}^{N} \mathbb{E}\left|\mathbb{E} H_{\varepsilon}\left(x-Z_{t_{l}}\right)\right|_{x=\bar{Z}_{t_{l}}^{i}}-\frac{1}{N} \sum_{j=1}^{N} H_{\varepsilon}\left(Y_{t_{l}}^{i}-Y_{t_{l}}^{j}\right) \mid+C\left(\frac{\sqrt{\varepsilon}}{\sqrt{\Delta t}}+\frac{1}{N}\right)$.

We choose $\varepsilon \leq \Delta t^{3}$. The next step consists in introducing the initialization error:

$$
\begin{aligned}
\bar{E}_{t_{l}} \leq & \frac{1}{N} \sum_{i=1}^{N} \mathbb{E}\left|\mathbb{E} H_{\varepsilon}\left(x-Z_{t_{l}}\right)\right|_{x=\bar{Z}_{t_{l}}^{i}}-\left.\frac{1}{N} \sum_{j=1}^{N} \mathbb{E} H_{\varepsilon}\left(x-Z_{t_{l}}^{0, y_{0}^{j}}\right)\right|_{x=\bar{Z}_{t_{l}}^{i}} \mid \\
& +\frac{1}{N} \sum_{i=1}^{N} \mathbb{E}\left|\frac{1}{N} \sum_{j=1}^{N}\left(\left.\mathbb{E} H_{\varepsilon}\left(x-Z_{t_{l}}^{0, y_{0}^{j}}\right)\right|_{x=\bar{Z}_{t_{l}}^{i}}-H_{\varepsilon}\left(Y_{t_{l}}^{i}-Y_{t_{l}}^{j}\right)\right)\right| \\
& +C\left(\Delta t+\frac{1}{N}\right) .
\end{aligned}
$$


Following the same technique as in the proof of Lemma 3.2. we have

$$
\begin{aligned}
& \frac{1}{N} \sum_{i=1}^{N} \mathbb{E}\left|\mathbb{E} H_{\varepsilon}\left(x-Z_{t_{l}}\right)\right|_{x=\bar{Z}_{t_{l}}^{i}}-\left.\frac{1}{N} \sum_{j=1}^{N} \mathbb{E} H_{\varepsilon}\left(x-Z_{t_{l}}^{0, y_{0}^{j}}\right)\right|_{x=\bar{Z}_{t_{l}}^{i}} \mid \\
& \quad \leq \sup _{x \in \mathbb{R}}\left|\int_{\mathbb{R}} \mathbb{E} H_{\varepsilon}\left(x-Z_{t}^{0, y}\right) m_{0}(d y)-\int_{\mathbb{R}} \mathbb{E} H_{\varepsilon}\left(x-Z_{t}^{0, y}\right) \bar{m}_{0}(d y)\right| \\
& \quad \leq C\left\|V_{0}-\bar{V}_{0}\right\|_{L^{\infty}(\mathbb{R})} .
\end{aligned}
$$

The third step consists in making apparent the error of the Euler scheme: for all $y \in \mathbb{R}$, we denote by $\left(\bar{Z}_{t_{k}}^{0, y}, k=0, \ldots, K\right)$ the discrete-time process solution of

$$
\left\{\begin{array}{l}
\bar{Z}_{t_{k+1}}^{0, y}=\bar{Z}_{t_{k}}^{0, y}+\Delta t B\left(t_{k}, \bar{Z}_{t_{k}}^{0, y}\right)+\sigma\left(W_{t_{k+1}}-W_{t_{k}}\right), \\
\bar{Z}_{0}^{0, y}=y
\end{array}\right.
$$

Then,

$$
\begin{aligned}
\bar{E}_{t_{l}} \leq & \frac{1}{N^{2}} \sum_{i, j=1}^{N}\left|\mathbb{E} H_{\varepsilon}\left(x-Z_{t_{l}}^{0, y_{0}^{j}}\right)\right|_{x=\bar{Z}_{t_{l}}^{i}}-\left.\mathbb{E} H_{\varepsilon}\left(x-\bar{Z}_{t_{l}}^{0, y_{0}^{j}}\right)\right|_{x=\bar{Z}_{t_{l}}^{i}} \mid \\
& +\frac{1}{N} \sum_{i=1}^{N} \mathbb{E}\left|\frac{1}{N} \sum_{j=1}^{N}\left(\left.\mathbb{E} H_{\varepsilon}\left(x-\bar{Z}_{t_{l}}^{0, y_{0}^{j}}\right)\right|_{x=\bar{Z}_{t_{l}}^{i}}-H_{\varepsilon}\left(Y_{t_{l}}^{i}-Y_{t_{l}}^{j}\right)\right)\right| \\
& +C\left(\Delta t+\frac{1}{N}+\left\|V_{0}-\bar{V}_{0}\right\|_{L^{\infty}(\mathbb{R})}\right) .
\end{aligned}
$$

In the right-hand side of the expression above, the first term is a time discretization error in the weak sense. It is described by the following lemma, the proof of which is postponed until the end of this subsection.

Lemma 3.11. Assume (H1), (H2) and (H3). For all $x$ and $y$ in $\mathbb{R}$ and all discrete time $t_{l}, l$ in $\{1, \ldots, K\}$,

$$
\left|\mathbb{E} H_{\varepsilon}\left(x-Z_{t_{l}}^{0, y}\right)-\mathbb{E} H_{\varepsilon}\left(x-\bar{Z}_{t_{l}}^{0, y}\right)\right| \leq C \Delta t,
$$

where the positive constant $C$ depends on $\sigma, V_{0}$ and $T$ only and is uniform in $x$ and $y$.

Thus,

$$
\begin{aligned}
\bar{E}_{t_{l} \leq} \leq & \frac{1}{N} \sum_{i=1}^{N} \mathbb{E}\left|\frac{1}{N} \sum_{j=1}^{N}\left(\left.\mathbb{E} H_{\varepsilon}\left(x-\bar{Z}_{t_{l}}^{0, y_{0}^{j}}\right)\right|_{x=\bar{Z}_{t_{l}}^{i}}-H_{\varepsilon}\left(Y_{t_{l}}^{i}-Y_{t_{l}}^{j}\right)\right)\right| \\
& +C\left(\Delta t+\frac{1}{N}+\left\|V_{0}-\bar{V}_{0}\right\|_{L^{\infty}(\mathbb{R})}\right) .
\end{aligned}
$$


We observe that $\bar{Z}_{t_{l}}^{0, y_{0}^{j}}$ and $\bar{Z}_{t_{l}}^{j}$ have the same law. In the last step, we introduce a statistical error:

$$
\begin{aligned}
\bar{E}_{t_{l}} \leq & \frac{1}{N} \sum_{i=1}^{N} \mathbb{E}\left|\frac{1}{N} \sum_{j=1}^{N}\left(\left.\mathbb{E} H_{\varepsilon}\left(x-\bar{Z}_{t_{l}}^{j}\right)\right|_{x=\bar{Z}_{t_{l}}^{i}}-H_{\varepsilon}\left(\bar{Z}_{t_{l}}^{i}-\bar{Z}_{t_{l}}^{j}\right)\right)\right| \\
& +\frac{1}{N^{2}} \sum_{i, j=1}^{N} \mathbb{E}\left|H_{\varepsilon}\left(\bar{Z}_{t_{l}}^{i}-\bar{Z}_{t_{l}}^{j}\right)-H_{\varepsilon}\left(Y_{t_{l}}^{i}-Y_{t_{l}}^{j}\right)\right| \\
& +C\left(\Delta t+\frac{1}{N}+\left\|V_{0}-\bar{V}_{0}\right\|_{L^{\infty}(\mathbb{R})}\right) .
\end{aligned}
$$

Let $\mathcal{F}_{t}^{i}:=\sigma\left(W_{s}^{i} ; 0 \leq s \leq t\right)$. For $j \neq i$ and with $\bar{Z}_{t_{l}}^{j}$ and $\bar{Z}_{t_{l}}^{j}$ independent, we have

$$
\mathbb{E}^{\mathcal{F}_{t_{l}}^{i}}\left(\left.\mathbb{E} H_{\varepsilon}\left(x-\bar{Z}_{t_{l}}^{j}\right)\right|_{x=\bar{Z}_{t_{l}}^{i}}-H_{\varepsilon}\left(\bar{Z}_{t_{l}}^{i}-\bar{Z}_{t_{l}}^{j}\right)\right)=0,
$$

which implies that

$$
\begin{aligned}
& \mathbb{E}\left(\frac{1}{N} \sum_{j=1 ; j \neq i}^{N}\left(\left.\mathbb{E} H_{\varepsilon}\left(x-\bar{Z}_{t_{l}}^{j}\right)\right|_{x=\bar{Z}_{t_{l}}^{i}}-H_{\varepsilon}\left(\bar{Z}_{t_{l}}^{i}-\bar{Z}_{t_{l}}^{j}\right)\right)\right)^{2} \\
& =\frac{1}{N^{2}} \sum_{j=1 ; j \neq i}^{N} \mathbb{E}\left(\left.\mathbb{E} H_{\varepsilon}\left(x-\bar{Z}_{t_{l}}^{j}\right)\right|_{x=\bar{Z}_{t_{l}}^{i}}-H_{\varepsilon}\left(\bar{Z}_{t_{l}}^{i}-\bar{Z}_{t_{l}}^{j}\right)\right)^{2} \leq \frac{2}{N} .
\end{aligned}
$$

Finally, we have obtained that

$$
\begin{aligned}
\bar{E}_{t_{l}} \leq & \frac{1}{N^{2}} \sum_{i, j ; i \neq j} \mathbb{E}\left|H_{\varepsilon}\left(\bar{Z}_{t_{l}}^{i}-\bar{Z}_{t_{l}}^{j}\right)-H_{\varepsilon}\left(Y_{t_{l}}^{i}-Y_{t_{l}}^{j}\right)\right| \\
& +C\left(\Delta t+\left\|V_{0}-\bar{V}_{0}\right\|_{L^{\infty}(\mathbb{R})}+\frac{1}{\sqrt{N}}\right) .
\end{aligned}
$$

It remains to analyze the term

$$
\frac{1}{N^{2}} \sum_{i \neq j} \mathbb{E}\left|H_{\varepsilon}\left(\bar{Z}_{t_{l}}^{i}-\bar{Z}_{t_{l}}^{j}\right)-H_{\varepsilon}\left(Y_{t_{l}}^{i}-Y_{t_{l}}^{j}\right)\right|
$$

in the right-hand side of (3.24). We do so by making apparent the successive transitions of the processes $\left(\bar{Z}^{i}\right)$ : for all $y$ in $\mathbb{R}$ and all $l$ in $\{0, \ldots, K\}$ we denote by $\left(\bar{Z}_{t_{k}}^{i, t_{l}, y}, k=l, \ldots, K\right)$ the discrete-time process solution of

$$
\left\{\begin{array}{l}
\bar{Z}_{t_{k+1}, t_{l}, y}^{i, 1}=\bar{Z}_{t_{k}}^{i, t_{l}, y}+\Delta t B\left(t_{k}, \bar{Z}_{t_{k}}^{i, t_{l}, y}\right)+\sigma\left(W_{t_{k+1}}^{i}-W_{t_{k}}^{i}\right), \\
\bar{Z}_{t_{l}, t_{l}, y}^{i}=y
\end{array}\right.
$$


Then,

$$
\begin{gathered}
\frac{1}{N^{2}} \sum_{i \neq j} \mathbb{E}\left|H_{\varepsilon}\left(\bar{Z}_{t_{l}}^{i}-\bar{Z}_{t_{l}}^{j}\right)-H_{\varepsilon}\left(Y_{t_{l}}^{i}-Y_{t_{l}}^{j}\right)\right| \\
\leq \sum_{n=0}^{l-1} \frac{1}{N^{2}} \sum_{i \neq j} \mathbb{E} \mid H_{\varepsilon}\left(\bar{Z}_{t_{l}}^{i, t_{l-n}, Y_{t_{l-n}}^{i}}-\bar{Z}_{t_{l}}^{j, t_{l-n}, Y_{t_{l-n}}^{j}}\right) \\
-H_{\varepsilon}\left(\bar{Z}_{t_{l}}^{i, t_{l-n-1}, Y_{t_{l-n-1}}^{i}}-\bar{Z}_{t_{l}}^{j, t_{l-n-1}, Y_{t_{l-n-1}}^{j}}\right) \mid .
\end{gathered}
$$

For each term in the sum over $n$, we use the identity

$$
H_{\varepsilon}(a)-H_{\varepsilon}(b)=(a-b) \int_{0}^{1} g_{\varepsilon}(b+u(a-b)) d u
$$

to get

$$
\begin{aligned}
& \frac{1}{N^{2}} \sum_{i \neq j} \mathbb{E} \mid H_{\varepsilon}\left(\bar{Z}_{t_{l}}^{i, t_{l-n}, Y_{t_{l-n}}^{i}}-\bar{Z}_{t_{l}}^{j, t_{l-n}, Y_{t_{l-n}}^{j}}\right)-H_{\varepsilon}\left(\bar{Z}_{t_{l}}^{i, t_{l-n-1}, Y_{t_{l-n-1}}^{i}}-\bar{Z}_{t_{l}}^{j, t_{l-n-1}, Y_{t_{l-n-1}}^{j}}\right) \mid \\
&=\frac{1}{N^{2}} \sum_{i \neq j} \mathbb{E} \mid\left[\left(\bar{Z}_{t_{l}}^{i, t_{l-n}, Y_{t_{l-n}}^{i}}-\bar{Z}_{t_{l}}^{i, t_{l-n-1}, Y_{t_{l-n-1}}^{i}}\right)\right. \\
&\left.-\left(\bar{Z}_{t_{l}, t_{l-n}, Y_{t_{l-n}}^{j}}^{j}-\bar{Z}_{t_{l}}^{j, t_{l-n-1}, Y_{t_{l-n-1}}^{j}}\right)\right] \\
& \times \int_{0}^{1} g_{\varepsilon}\left(R_{t_{l}, u}^{i, t_{l-n-1}}-R_{t_{l}, u}^{j, t_{l-n-1}}\right) d u \mid,
\end{aligned}
$$

where, for any $i$ in $\{1, \ldots, N\}$, we define the random variables $R_{t_{l}, u}^{i, t_{l-n-1}}$ by

$$
R_{t_{l}, u}^{i, t_{l-n-1}}:=\bar{Z}_{t_{l}}^{i, t_{l-n-1}, Y_{t_{l-n-1}}^{i}}+u\left(\bar{Z}_{t_{l}}^{i, t_{l-n}, Y_{t_{l-n}}^{i}}-\bar{Z}_{t_{l}}^{i, t_{l-n-1}, Y_{t_{l-n-1}}^{i}}\right) .
$$

As the drift $B(t, x)$ of $\left(\bar{Z}^{i}\right)$ is a Lipschitz function, one can easily show that, for any $i$ in $\{0, \ldots, N\}$,

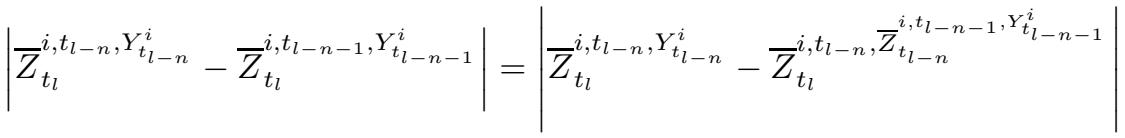

$$
\begin{aligned}
& \leq C\left|Y_{t_{l-n}}^{i}-\bar{Z}_{t_{l-n}}^{i, t_{l-n-1}, Y_{t_{l-n-1}}^{i}}\right|
\end{aligned}
$$

and hence that

$$
\begin{aligned}
& \left|\bar{Z}_{t_{l}}^{i, t_{l-n}, Y_{t_{l-n}}^{i}}-\bar{Z}_{t_{l}}^{i, t_{l-n-1}, Y_{t_{l-n-1}}^{i}}\right| \\
& \quad \leq C \Delta t\left|\bar{V}_{t_{l-n-1}}\left(Y_{t_{l-n-1}}^{i}\right)-V\left(t_{l-n-1}, Y_{t_{l-n-1}}^{i}\right)\right| .
\end{aligned}
$$


Then, we have

$$
\begin{aligned}
& \frac{1}{N^{2}} \sum_{i \neq j} \mathbb{E} \mid H_{\varepsilon}\left(\bar{Z}_{t_{l}}^{i, t_{l-n}, Y_{t_{l-n}}^{i}}-\bar{Z}_{t_{l}}^{\left.j, t_{l-n}, Y_{t_{l-n}}^{j}\right)}\right. \\
&-H_{\varepsilon}\left(\bar{Z}_{t_{l}}^{i, t_{l-n-1}, Y_{t_{l-n-1}}^{i}}-\bar{Z}_{t_{l}}^{j, t_{l-n-1}, Y_{t_{l-n-1}}^{j}}\right) \mid \\
& \leq C \Delta t \frac{1}{N^{2}} \sum_{i \neq j} \mathbb{E} \mid\left(\mid \bar{V}_{t_{l-n-1}}\left(Y_{t_{l-n-1}}^{i}\right)-\right. V\left(t_{l-n-1}, Y_{t_{l-n-1}}^{i}\right) \mid \\
&\left.+\left|\bar{V}_{t_{l-n-1}}\left(Y_{t_{l-n-1}}^{j}\right)-V\left(t_{l-n-1}, Y_{t_{l-n-1}}^{j}\right)\right|\right) \\
& \times \int_{0}^{1} g_{\varepsilon}\left(R_{t_{l}, u}^{i, t_{l-n-1}}-R_{t_{l}, u}^{j, t_{l-n-1}}\right) d u \mid .
\end{aligned}
$$

We introduce the conditional expectation with respect to $\mathcal{F}_{t_{l-n-1}}$ in the right-hand side of the expression above. As, for any $i \geq 1, \mid \bar{V}_{t_{l-n-1}}\left(Y_{t_{l-n-1}}^{i}\right)-$ $V\left(t_{l-n-1}, Y_{t_{l-n-1}}^{i}\right) \mid$ is an $\mathcal{F}_{t_{l-n-1}}$-measurable variable, we obtain that

$$
\begin{aligned}
& \mathbb{E} \mid H_{\varepsilon}\left(\bar{Z}_{t_{l}}^{i, t_{l-n}, Y_{t_{l-n}}^{i}}-\bar{Z}_{t_{l}}^{j, t_{l-n}, Y_{t_{l-n}}^{j}}\right) \\
&-H_{\varepsilon}\left(\bar{Z}_{t_{l}}^{i, t_{l-n-1}, Y_{t_{l-n-1}}^{i}}-\bar{Z}_{t_{l}}^{j, t_{l-n-1}, Y_{t_{l-n-1}}^{j}}\right) \mid \\
& \leq C \Delta t \mathbb{E} \mid\left(\left|\bar{V}_{t_{l-n-1}}\left(Y_{t_{l-n-1}}^{i}\right)-V\left(t_{l-n-1}, Y_{t_{l-n-1}}^{i}\right)\right|\right. \\
&\left.+\left|\bar{V}_{t_{l-n-1}}\left(Y_{t_{l-n-1}}^{j}\right)-V\left(t_{l-n-1}, Y_{t_{l-n-1}}^{j}\right)\right|\right) \\
& \times \int_{0}^{1} \mathbb{E}^{\mathcal{F}_{t_{l-n-1}}}\left\{g_{\varepsilon}\left(R_{t_{l}, u}^{i, t_{l-n-1}}-R_{t_{l}, u}^{j, t_{l-n-1}}\right)\right\} d u \mid .
\end{aligned}
$$

Now, we need to bound $\mathbb{E}^{\mathcal{F}_{t_{l-n-1}}}\left\{g_{\varepsilon}\left(R_{t_{l}, u}^{i, t_{l-n-1}}-R_{t_{l}, u}^{j, t_{l-n-1}}\right)\right\}$. Coming back to the definition of $R_{t_{l}, u}^{i, t_{l-n-1}}$ in (3.27) and using the equation (3.25) satisfied by $\left(\bar{Z}_{t_{k}}^{i, t_{l}, y}, k=l, \ldots, K\right)$, we remark that

$$
R_{t_{l}, u}^{i, t_{l-n-1}}=Y_{t_{l-n-1}}^{i}+\sigma\left(W_{t_{l}}^{i}-W_{t_{l-n-1}}^{i}\right)+\int_{t_{l-n-1}}^{t_{l}} \psi_{u}^{i}(\theta) d \theta
$$

where, for all $\theta \in\left[t_{l-n-1}, T\right]$,

$$
\begin{aligned}
\psi_{u}^{i}(\theta)= & u A^{\prime}\left(\bar{V}_{t_{l-n-1}}\left(Y_{t_{l-n-1}}^{i}\right)\right) \mathbb{1}_{\left[t_{l-n-1}, t_{l-n}[\right.}(\theta) \\
& +\sum_{k=l-n}^{K} u B\left(t_{k}, \bar{Z}_{t_{k}}^{i, t_{l-n}, Y_{t_{l-n}}^{i}}\right) \mathbb{1}_{\left[t_{k}, t_{k+1}[\right.}(\theta) \\
& +\sum_{k=l-n-1}^{K}(1-u) B\left(t_{k}, \bar{Z}_{t_{k}}^{\left.i, t_{l-n-1}, Y_{t_{l-n-1}}^{i}\right)} \mathbb{1}_{\left[t_{k}, t_{k+1}[\right.}(\theta) .\right.
\end{aligned}
$$


For $i \neq j$, conditionally on $\mathcal{F}_{t_{l-n-1}}$, for any $k>l-n-1, \bar{Z}_{t_{k}}^{i, t_{l-n-1}, Y_{t_{l-n-1}}^{i}}$ and $\bar{Z}_{t_{k}}^{j, t_{l-n-1}, Y_{t_{l-n-1}}^{j}}$ are independent, as well as $\bar{Z}_{t_{k}}^{i, t_{l-n}, Y_{t_{l-n}}^{i}}$ and $\bar{Z}_{t_{k}}^{j, t_{l-n}, Y_{t_{l-n}}^{j}}$. Therefore, $R_{t_{l}, u}^{i, t_{l-n-1}}$ and $R_{t_{l}, u}^{j, t_{l-n-1}}$ are independent conditionally on $\mathcal{F}_{t_{l-n-1}}$. Moreover, with $\psi_{u}^{i}(\theta)$ uniformly bounded, by the Girsanov theorem, the law of $R_{t_{l}, u}^{i, t_{l-n-1}}$ conditionally on $\mathcal{F}_{t_{l-n-1}}$ has a density denoted by $\widetilde{\Gamma}\left(t_{l}, \cdot ; t_{l-n-1}, Y_{t_{l-n-1}}^{i}\right)$. Applying $\operatorname{Remark}$ 3.5. $\widetilde{\Gamma}\left(t_{l}, \cdot ; t_{l-n-1}, Y_{t_{l-n-1}}^{i}\right)$ is in $L^{2}(\mathbb{R})$ and

$$
\left\|\widetilde{\Gamma}\left(t_{l}, \cdot ; t_{l-n-1}, Y_{t_{l-n-1}}^{i}\right)\right\|_{L^{2}(\mathbb{R})} \leq \frac{C}{t_{n+1}^{1 / 4}} .
$$

Thus, for $i \neq j$,

$$
\begin{aligned}
& \mathbb{E}^{\mathcal{F}_{t_{l-n-1}}}\left\{g_{\varepsilon}\left(R_{t_{l}, u}^{i, t_{l-n-1}}-R_{t_{l}, u}^{j, t_{l-n-1}}\right)\right\} \\
& \quad=\int_{\mathbb{R}} \int_{\mathbb{R}} g_{\varepsilon}(z-y) \widetilde{\Gamma}\left(t_{l}, z ; t_{l-n-1}, Y_{t_{l-n-1}}^{i}\right) \widetilde{\Gamma}\left(t_{l}, y ; t_{l-n-1}, Y_{t_{l-n-1}}^{j}\right) d z d y \\
& \quad \leq\left\|\widetilde{\Gamma}\left(t_{l}, \cdot ; t_{l-n-1}, Y_{t_{l-n-1}}^{i}\right)\right\|_{L^{2}(\mathbb{R})}\left\|\widetilde{\Gamma}\left(t_{l}, \cdot ; t_{l-n-1}, Y_{t_{l-n-1}}^{j}\right)\right\|_{L^{2}(\mathbb{R})} \\
& \quad \leq \frac{C}{\sqrt{t_{n+1}}}
\end{aligned}
$$

Combining this last upper bound with (3.29), we obtain that

$$
\begin{aligned}
& \frac{1}{N^{2}} \sum_{i \neq j} \mathbb{E} \mid H_{\varepsilon}\left(\bar{Z}_{t_{l}}^{i, t_{l-n}, Y_{t_{l-n}}^{i}}-\bar{Z}_{t_{l}}^{j, t_{l-n}, Y_{t_{l-n}}^{j}}\right) \\
& \quad-H_{\varepsilon}\left(\bar{Z}_{t_{l}}^{i, t_{l-n-1}, Y_{t_{l-n-1}}^{i}}-\bar{Z}_{t_{l}}^{j, t_{l-n-1}, Y_{t_{l-n-1}}^{j}}\right) \mid \\
& \leq \frac{C \Delta t}{\sqrt{t_{n+1}}} \frac{1}{N} \sum_{i=1}^{N} \mathbb{E}\left|\bar{V}_{t_{l-n-1}}\left(Y_{t_{l-n-1}}^{i}\right)-V\left(t_{l-n-1}, Y_{t_{l-n-1}}^{i}\right)\right|
\end{aligned}
$$

and using (3.23), that

$$
\begin{aligned}
& \frac{1}{N^{2}} \sum_{i \neq j} \mathbb{E} \mid H_{\varepsilon}\left(\bar{Z}_{t_{l}}^{i, t_{l-n}, Y_{t_{l-n}}^{i}}-\bar{Z}_{t_{l}}^{j, t_{l-n}, Y_{t_{l-n}}^{j}}\right) \\
& \quad-H_{\varepsilon}\left(\bar{Z}_{t_{l}}^{i, t_{l-n-1}, Y_{t_{l-n-1}}^{i}}-\bar{Z}_{t_{l}}^{j, t_{l-n-1}, Y_{t_{l-n-1}}^{j}}\right) \mid \\
& \leq \frac{C \Delta t}{\sqrt{t_{n+1}}}\left(\bar{E}_{t_{l-n-1}}+C \Delta t \sum_{m=0}^{l-n-2} \bar{E}_{t_{m}}\right) .
\end{aligned}
$$


As in (3.26), we sum the term above over $n$ in $\{0, \ldots, l-1\}$ to finally obtain that

$$
\begin{aligned}
& \frac{1}{N^{2}} \sum_{i \neq j} \mathbb{E}\left|H_{\varepsilon}\left(\bar{Z}_{t_{l}}^{i}-\bar{Z}_{t_{l}}^{j}\right)-H_{\varepsilon}\left(Y_{t_{l}}^{i}-Y_{t_{l}}^{j}\right)\right| \\
& \leq \sum_{n=0}^{l-1}\left(\frac{C \Delta t}{\sqrt{t_{n+1}}}\left(\bar{E}_{t_{l-n-1}}+C \Delta t \sum_{m=0}^{l-n-2} \bar{E}_{t_{m}}\right)\right) \\
& \quad \leq \sum_{n=0}^{l-1} \frac{C \Delta t}{\sqrt{t_{n+1}}} \bar{E}_{t_{l-n-1}}+\sum_{m=0}^{l-1} C \Delta t \bar{E}_{t_{m}} .
\end{aligned}
$$

This last bound, combined with (3.24), gives the induction relation

$$
\bar{E}_{t_{l}} \leq \sum_{n=0}^{l-1} \frac{C \Delta t}{\sqrt{t_{l}-t_{n}}} \bar{E}_{t_{n}}+C\left(\Delta t+\left\|V_{0}-\bar{V}_{0}\right\|_{L^{\infty}(\mathbb{R})}+\frac{1}{\sqrt{N}}\right) .
$$

Proof of Lemma 3.11. To study this weak type error for the Euler scheme, we follow a technique due to Talay and Tubaro [9]. The main idea consists in using the Feynman-Kac representation of a Cauchy problem and noting that $\mathbb{E} H_{\varepsilon}\left(x-Z_{t_{l}}^{0, y}\right)=v_{t_{l}, x}(0, y)$, where the function $v_{t_{l}, x}(s, y)$ is the solution of the partial differential equation

$$
\left\{\begin{array}{c}
\frac{\partial v_{t_{l}, x}}{\partial s}(s, y)+\frac{1}{2} \sigma^{2} \frac{\partial^{2} v_{t_{l}, x}}{\partial y^{2}}(s, y)+B(s, y) \frac{\partial v_{t_{l}, x}}{\partial y}(s, y)=0 \\
\forall(s, y) \in\left[0, t_{l}\right) \times \mathbb{R} \\
v_{t_{l}, x}\left(t_{l}, y\right)=H_{\varepsilon}(x-y), \forall y \in \mathbb{R}
\end{array}\right.
$$

The above Cauchy problem is similar to (3.6) and the results of Lemma 3.9 hold for (3.30), replacing $t_{k}$ by $t_{l}$ in the setting. Thus

$$
\mathbb{E} H_{\varepsilon}\left(x-Z_{t_{l}}^{0, y}\right)-\mathbb{E} H_{\varepsilon}\left(x-\bar{Z}_{t_{l}}^{0, y}\right)=v_{t_{l}, x}(0, y)-\mathbb{E} v_{t_{l}, x}\left(t_{l}, \bar{Z}_{t_{l}}^{0, y}\right) .
$$

In the sequel, we will use the notation $v$ rather than $v_{t_{l}, x}$, except when we need to make apparent the parameters $x$ and $t_{l}$. We decompose the expression above, making apparent the discrete dates in $\left[0, t_{l}\right)$ :

$$
v_{t_{l}, x}(0, y)-\mathbb{E} v_{t_{l}, x}\left(t_{l}, \bar{Z}_{t_{l}}^{0, y}\right)=-\sum_{n=0}^{l-1} \mathbb{E}\left(v\left(t_{n+1}, \bar{Z}_{t_{n+1}}^{0, y}\right)-v\left(t_{n}, \bar{Z}_{t_{n}}^{0, y}\right)\right) .
$$

We apply Itô's formula for the first time and use (3.30) to obtain $v_{t_{l}, x}(0, y)-\mathbb{E} v_{t_{l}, x}\left(t_{l}, \bar{Z}_{t_{l}}^{0, y}\right)=\sum_{n=0}^{l-1} \mathbb{E} \int_{t_{n}}^{t_{n+1}} \frac{\partial v}{\partial y}\left(s, \bar{Z}_{s}^{0, y}\right)\left(B\left(s, \bar{Z}_{s}^{0, y}\right)-B\left(t_{n}, \bar{Z}_{t_{n}}^{0, y}\right)\right) d s$, where $\bar{Z}_{s}^{0, y}=\bar{Z}_{t_{n}}^{0, y}+s B\left(t_{n}, \bar{Z}_{t_{n}}^{0, y}\right)+\sigma\left(W_{s}-W_{t_{n}}\right)$ when $s \in\left[t_{n}, t_{n+1}\right)$. Applying the 
Itô formula and (3.30) again,

$$
\begin{aligned}
& \mathbb{E} \int_{t_{n}}^{t_{n+1}} \frac{\partial v}{\partial y}\left(s, \bar{Z}_{s}^{0, y}\right)\left(B\left(s, \bar{Z}_{s}^{0, y}\right)-B\left(t_{n}, \bar{Z}_{t_{n}}^{0, y}\right)\right) d s \\
&=\mathbb{E} \int_{t_{n}}^{t_{n+1}} \int_{t_{n}}^{s}\left[\frac{\partial}{\partial \theta}+B\left(t_{n}, \bar{Z}_{t_{n}}^{0, y}\right) \frac{\partial}{\partial y}+\frac{\sigma^{2}}{2} \frac{\partial^{2}}{\partial y^{2}}\right] \quad \times\left(\frac{\partial v}{\partial y}\left(\theta, \bar{Z}_{\theta}^{0, y}\right)\left(B\left(\theta, \bar{Z}_{\theta}^{0, y}\right)-B\left(t_{n}, \bar{Z}_{t_{n}}^{0, y}\right)\right)\right) d \theta d s \\
&=\mathbb{E} \int_{t_{n}}^{t_{n+1}} \int_{t_{n}}^{s}\left[\frac { \partial v } { \partial y } ( \theta , \overline { Z } _ { \theta } ^ { 0 , y } ) \left(\frac{\partial B}{\partial \theta}\left(\theta, \bar{Z}_{\theta}^{0, y}\right)\right.\right.\left.\left.+\frac{\sigma^{2}}{2} \frac{\partial^{2} B}{\partial y^{2}}\left(\theta, \bar{Z}_{\theta}^{0, y}\right)\right)\right] d \theta d s \\
&+\mathbb{E} \int_{t_{n}}^{t_{n+1}} \int_{t_{n}}^{s}\left[\frac{\partial^{2} v}{\partial y^{2}}\left(\theta, \bar{Z}_{\theta}^{0, y}\right)\right. \\
&\left.\times\left(\sigma^{2} \frac{\partial B}{\partial y}\left(\theta, \bar{Z}_{\theta}^{0, y}\right)-\left(B\left(\theta, \bar{Z}_{\theta}^{0, y}\right)-B\left(t_{n}, \bar{Z}_{t_{n}}^{0, y}\right)\right)^{2}\right)\right] d \theta d s .
\end{aligned}
$$

Using the bounds on $B$ and its derivatives given in Lemma 3.6 we get

$$
\begin{aligned}
& \mathbb{E} H_{\varepsilon}\left(x-Z_{t_{l}}^{0, y}\right)-\mathbb{E} H_{\varepsilon}\left(x-\bar{Z}_{t_{l}}^{0, y}\right) \\
& \quad \leq C \sum_{n=0}^{l-1} \int_{t_{n}}^{t_{n+1}} \int_{t_{n}}^{s}\left(\mathbb{E}\left|\frac{\partial^{2} v}{\partial y^{2}}\left(\theta, \bar{Z}_{\theta}^{0, y}\right)\right|+\mathbb{E}\left|\frac{\partial v}{\partial y}\left(\theta, \bar{Z}_{\theta}^{0, y}\right)\right|\right) d \theta d s .
\end{aligned}
$$

Using the same technique as in the computation of (3.20), we obtain that

$$
\mathbb{E}\left|\frac{\partial v_{t_{l}, x}}{\partial y}\left(\theta, \bar{Z}_{\theta}^{0, y}\right)\right| \leq \frac{C}{\sqrt{t_{l}-\theta}} \quad \text { and } \quad \mathbb{E}\left|\frac{\partial^{2} v_{t_{l}, x}}{\partial y^{2}}\left(\theta, \bar{Z}_{\theta}^{0, y}\right)\right| \leq \frac{C}{\theta^{4 / 10}\left(t_{l}-\theta\right)^{3 / 5}},
$$

where the constant $C$ is uniform in $x$ and $y$. We integrate in time in (3.31) to get

$$
\left|\mathbb{E} H_{\varepsilon}\left(x-Z_{t_{l}}^{0, y}\right)-\mathbb{E} H_{\varepsilon}\left(x-\bar{Z}_{t_{l}}^{0, y}\right)\right| \leq C \Delta t
$$

\section{Conclusions}

In this paper, we have analyzed the rate of convergence of a stochastic particle method for one-dimensional viscous scalar conservation laws and showed that the rate of convergence is of order $\mathcal{O}(\Delta t+1 / \sqrt{N})$. This result is optimal in the sense that it is observed on numerical experiments when one applies the algorithm on the test case of the Burgers equation (see [3]).

The analysis of the algorithm with respect to the time step $\Delta t$ is based upon the analysis of the weak convergence of the Euler scheme. The techniques applied let us expect that it is possible to expand the discretization error in powers of the discretization step size $\Delta t$ at least up to the order two.

In the case of stochastic differential equations that are linear in the sense of McKean, such an expansion was initially showed by Talay and Tubaro [9]. The 
expansion up to the order two permits us to justify the use of the Romberg extrapolation which provides a second order accuracy with respect to the time step $\Delta t$.

Here, we simulated a nonlinear stochastic differential equation to compute the numerical solution of a nonlinear PDE. The nonlinearity of the SDE implies the simulation of a particle system. Even in this nonlinear case, it must be possible to adapt the Romberg extrapolation as a speed-up procedure.

Figures 14 present numerical experiments on the Burgers equation (1.2). We compare the numerical solution obtained with the present version of the particle method (for a given time step $\Delta t$ ) and a solution obtained by extrapolation between the solutions computed for the time steps $\Delta t$ and $\Delta t / 2$. More precisely, for a given $\Delta t$, let $\left(Y_{t_{k}}^{i, \Delta t}, i=1, \ldots, N ; k=0, \ldots, K\right)$ be the family of discrete time processes involved in the algorithm and defined in (2.3). We denote by $\bar{V}_{t_{k}}^{\Delta t}(x)$ the corresponding numerical solution defined in (2.4). For final time $T=K \Delta t$, we define the extrapolated solution $\bar{V}_{T}^{\Delta t, \Delta t / 2}(x)$ by

$$
\bar{V}_{T}^{\Delta t, \Delta t / 2}(x)=2 \bar{V}_{T}^{\Delta t / 2}(x)-\bar{V}_{T}^{\Delta t}(x) .
$$

If we are able to expand the error as

$$
\bar{V}_{T}^{\Delta t}(x)-V(T, x)=C(x) \Delta t+\mathcal{O}\left(\Delta t^{2}\right)+R(\omega),
$$

where the constant $C(x)$ does not depend on $\Delta t$ and where the random variable $R$ is such that $\mathbb{E}\|R\| \leq C^{\prime} / \sqrt{N}$ for an appropriate choice of the norm \|\| , then we will be in a position to conclude that $\mathbb{E}\left\|\bar{V}_{T}^{\Delta t, \Delta t / 2}(x)-V(T, x)\right\|$ is of order $\mathcal{O}\left(\Delta t^{2}+1 / \sqrt{N}\right)$. In the point of view of numerical tests, Figures 1 2 and 3 give encouraging results. But we can observe strong local error when we increase the time step $\Delta t$ (see Figure 3 for $\Delta t=0.01$ and Figure 4 for $\Delta t=0.05$ ). The sensibility on $\Delta t$ varies according to the choice of the initial condition and the viscosity parameter $\sigma$. This phenomenon can be easily explained. The constants in the expansion (4.2) must depend on the space variable $x$ and also on the derivatives of the solution $V$. This means that we need to choose $\Delta t$ sufficiently small to have $C(x) \Delta t$ large enough compared to $\Delta t^{2}$ for all $x$ and to benefit from the extrapolation procedure at all points $x$.

Moreover, the direct extrapolation procedure does not conserve the nature of the measure derivative of the corresponding numerical solution $\bar{V}_{T}^{\Delta t, \Delta t / 2}(x)$. For example, if the solution $V(T, x)$ is the distribution function of a probability measure, the same is true for the numerical solutions $\bar{V}_{T}^{\Delta t}(x)$ and $\bar{V}_{T}^{\Delta t / 2}(x)$ but not for $\bar{V}_{T}^{\Delta t, \Delta t / 2}(x)$. This is why in Figure 4 the extrapolated solution is a nonmonotonous function.

Thus we need to explore some variants of the direct extrapolation in order to reduce these phenomena. A tentative move in this direction could be based on the use of the extrapolation procedure during the computation, in order to correct the drifts of the particles, and not just at the final time. 

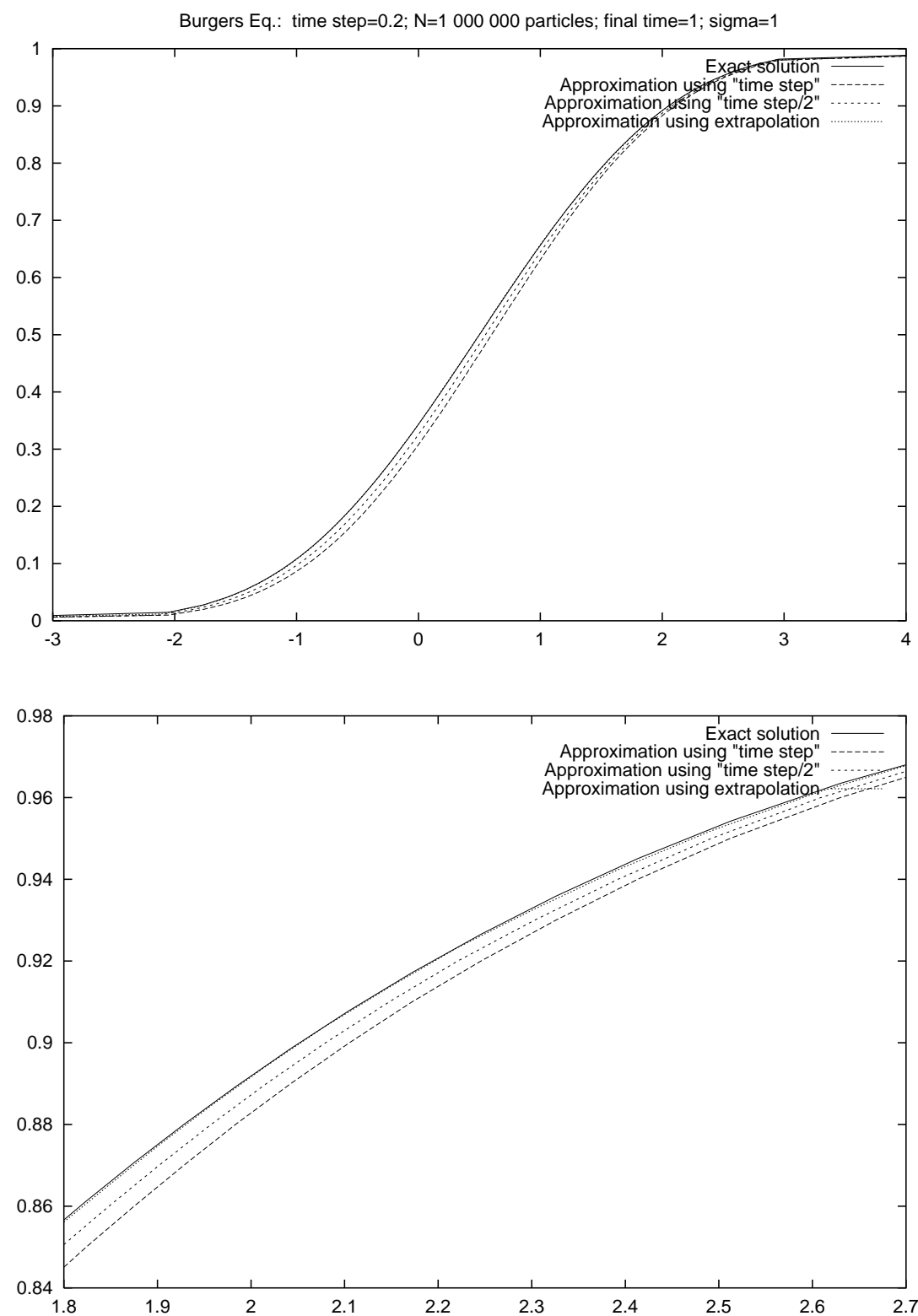

FiguRE 1. Exact and numerical solutions of the Burgers equation with initial condition $V(0, x)=H(x)$. The second picture shows a zoom for $x \in[1.8,2.7]$. The corresponding approximations of the $L^{1}$-norm of the error are $\left\|V(1, x)-\bar{V}_{1}^{\Delta t}(x)\right\|_{L^{1}(\mathbb{R})}=$ 0.0991, $\left\|V(1, x)-\bar{V}_{1}^{\Delta t / 2}(x)\right\|_{L^{1}(\mathbb{R})}=0.0501, \| V(1, x)-2 \bar{V}_{1}^{\Delta t / 2}(x)+$ $\bar{V}_{1}^{\Delta t}(x) \|_{L^{1}(\mathbb{R})}=0.00292$. 

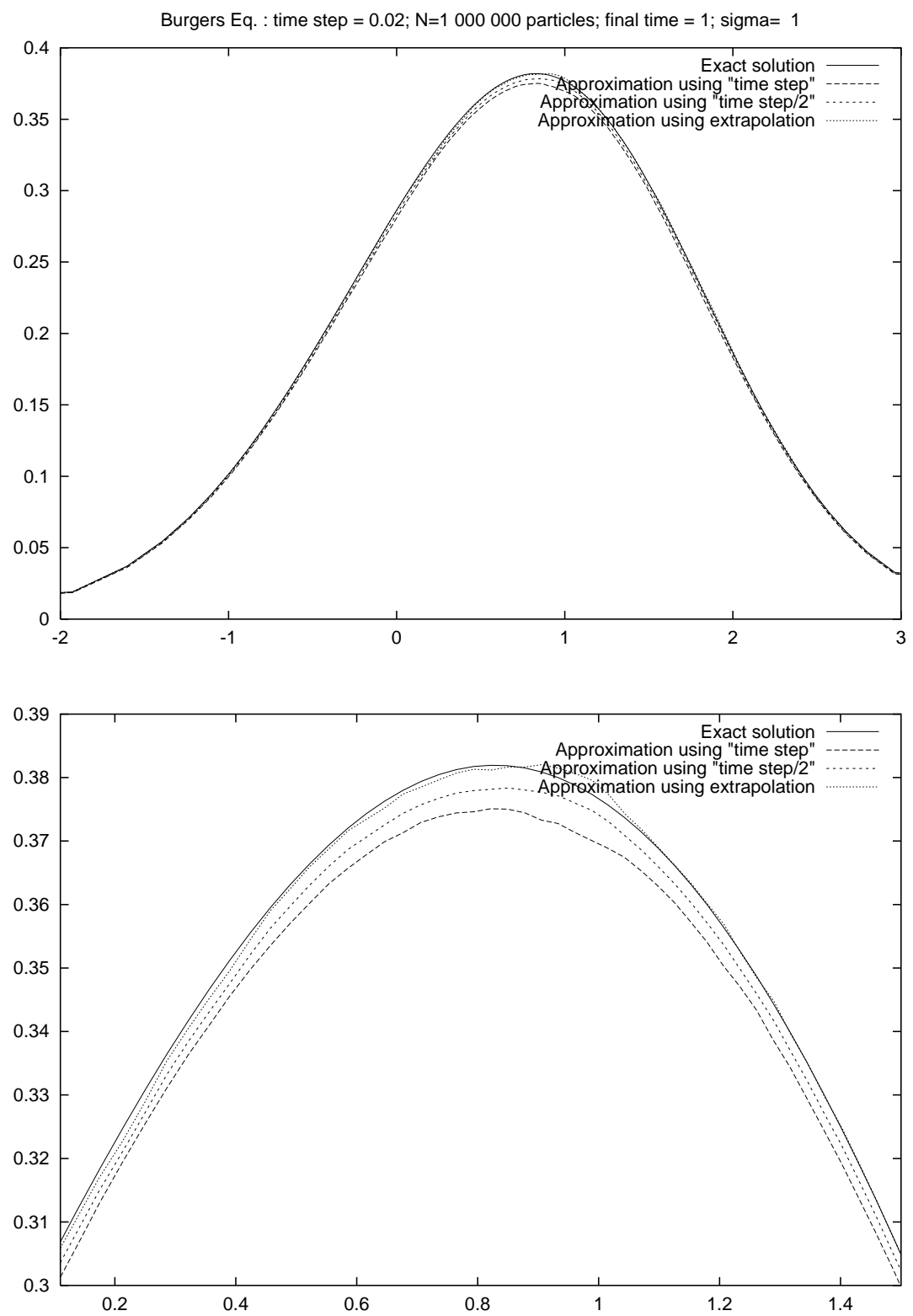

Figure 2. Exact and numerical solutions of the Burgers equation with initial condition $V(0, x)=H(x)-H(x-1)$. The second picture shows a zoom for $x \in[0.11,1.5]$. The corresponding approximations of the $L^{1}$-norm of the error are $\| V(1, x)-$ $\bar{V}_{1}^{\Delta t}(x)\left\|_{L^{1}(\mathbb{R})}=0.0183,\right\| V(1, x)-\bar{V}_{1}^{\Delta t / 2}(x) \|_{L^{1}(\mathbb{R})}=0.0094$, $\left\|V(1, x)-2 \bar{V}_{1}^{\Delta t / 2}(x)+\bar{V}_{1}^{\Delta t}(x)\right\|_{L^{1}(\mathbb{R})}=0.0030$. 

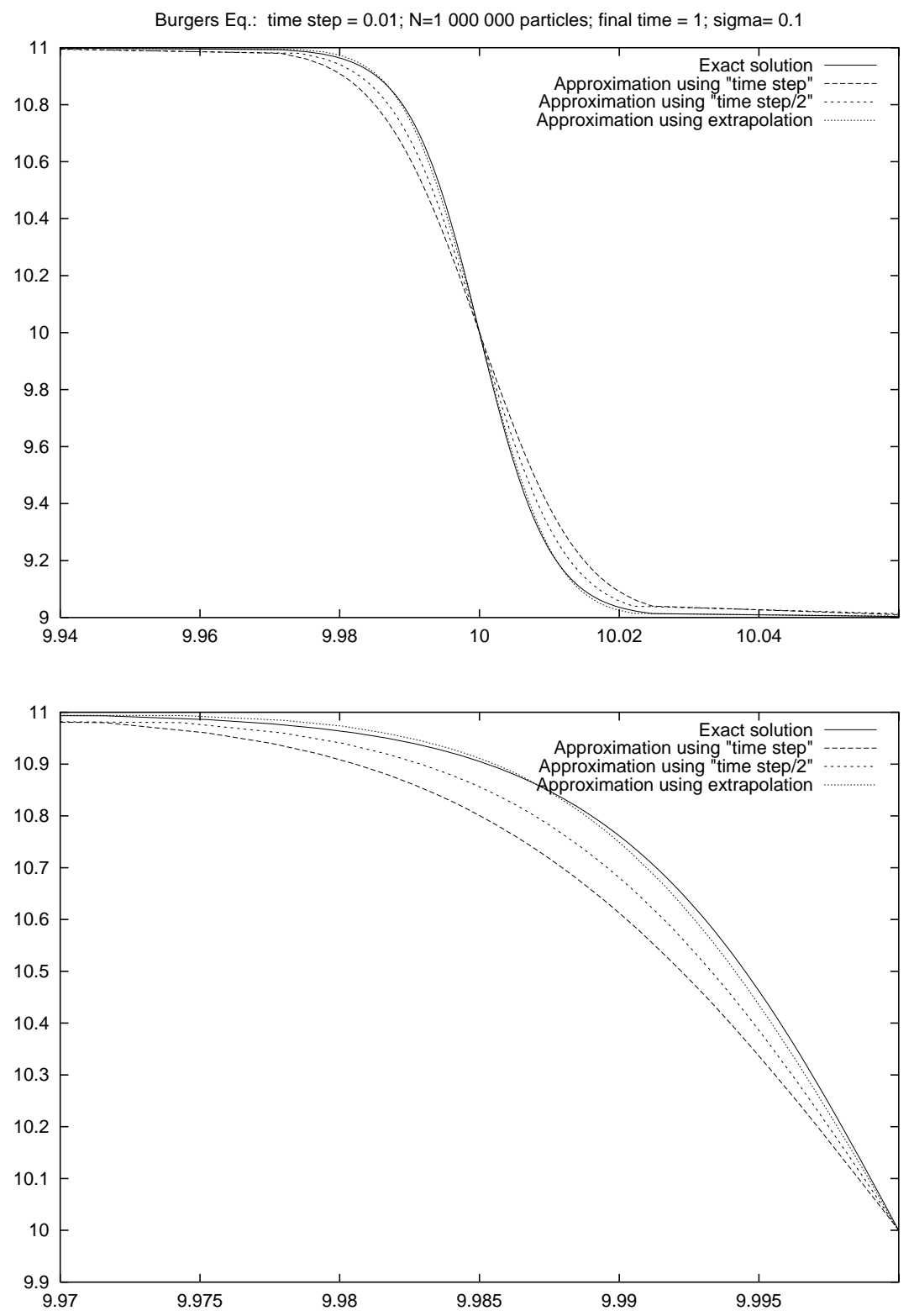

FiguRE 3. Exact and numerical solutions of the Burgers equation with initial condition $V(0, x)=10-\tanh \left(\frac{x}{\sigma^{2}}\right)$. The second picture shows a zoom for $x \in[9.97,10]$. The corresponding approximations of the $L^{1}$-norm of the error are $\left\|V(1, x)-\bar{V}_{1}^{\Delta t}(x)\right\|_{L^{1}(\mathbb{R})}=$ $0.0049,\left\|V(1, x)-\bar{V}_{1}^{\Delta t / 2}(x)\right\|_{L^{1}(\mathbb{R})}=0.0024, \| V(1, x)-2 \bar{V}_{1}^{\Delta t / 2}(x)+$ $\bar{V}_{1}^{\Delta t}(x) \|_{L^{1}(\mathbb{R})}=0.00062$. 

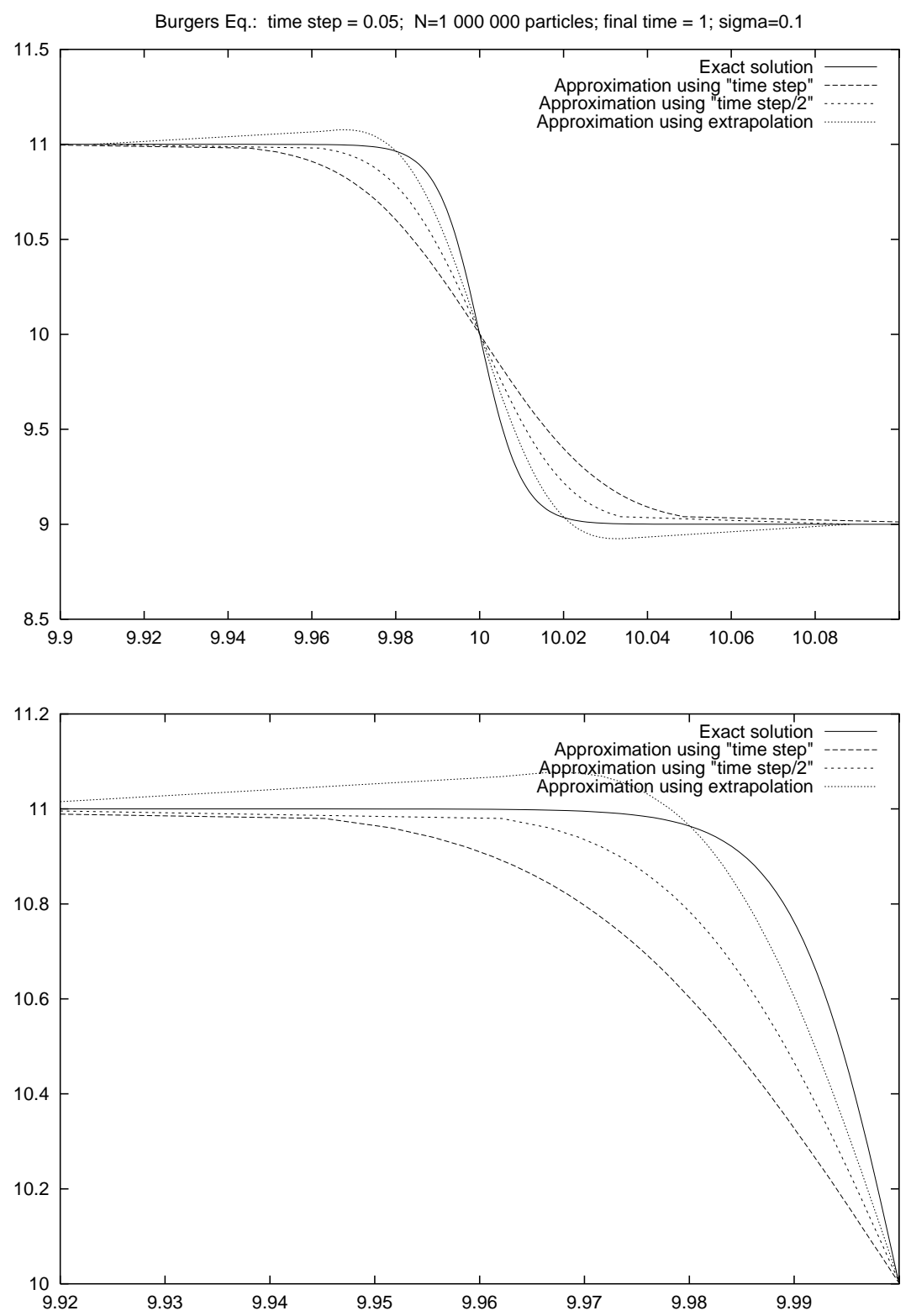

FiguRE 4. Exact and numerical solutions of the Burgers equation with initial condition $V(0, x)=10-\tanh \left(\frac{x}{\sigma^{2}}\right)$. The second picture shows a zoom for $x \in[9.92,10]$. The corresponding approximations of the $L^{1}$-norm of the error are $\left\|V(1, x)-\bar{V}_{1}^{\Delta t}(x)\right\|_{L^{1}(\mathbb{R})}=$ 0.024, $\left\|V(1, x)-\bar{V}_{1}^{\Delta t / 2}(x)\right\|_{L^{1}(\mathbb{R})}=0.012, \| V(1, x)-2 \bar{V}_{1}^{\Delta t / 2}(x)+$ $\bar{V}_{1}^{\Delta t}(x) \|_{L^{1}(\mathbb{R})}=0.0081$. 


\section{REFERENCES}

1. M. Bossy, L. Fezoui, and S. Piperno. Comparison of a stochastic particle method and a finite volume deterministic method applied to Burgers equation. Monte Carlo Methods and Appl., 3(2):113-140, 1997. MR 98f:65008

2. M. Bossy and D. Talay. Convergence rate for the approximation of the limit law of weakly interacting particles: application to the Burgers equation. Ann. Appl. Probab., 6:818-861, 1996. MR 97k:60158

3. M. Bossy and D. Talay. A stochastic particle method for the McKean-Vlasov and the Burgers equation. Math. Comp., 66(217):157-192, 1997. MR 97c:60233

4. A. Friedman. Partial Differential Equations of Parabolic Type. Prentice Hall, 1964. MR 31:6062

5. A. Friedman. Stochastic Differential Equations and Applications, volume 1. Academic Press, New York, 1975. MR 58:13350a

6. B. Jourdain. Diffusion processes associated with nonlinear evolution equations for signed measures. Methodology and Computing in Applied Probability, 2(1):69-91, April 2000. MR 2001f:60112

7. A. Kohatsu-Higa and S. Ogawa. Weak rate of convergence for a Euler scheme of nonlinear sde's. Monte Carlo Methods and Appl., 3:327-345, 1997. MR 98i:60053

8. S. Méléard and S. Roelly-Coppoletta. A propagation of chaos result for a system of particles with moderate interaction. Stochastic Proc. Appl., 26:317-332, 1987. MR 89e:60201

9. D. Talay and L. Tubaro. Expansion of the global error for numerical schemes solving stochastic differential equations. Stoch. Anal. Appl., 8(4):94-120, 1990. MR 92e:60124

INRIA, 2004 Route des Lucioles, B.P. 93, 06902 Sophia-Antipolis Cedex, France

E-mail address: Mireille.Bossy@sophia.inria.fr 